\title{
Re-evaluation of the traditional diet-heart hypothesis: analysis of recovered data from Minnesota Coronary Experiment (1968-73)
}

\author{
Christopher E Ramsden, ${ }^{1,2}$ Daisy Zamora, ${ }^{3}$ Sharon Majchrzak-Hong, ${ }^{1}$ Keturah R Faurot, ${ }^{2}$ \\ Steven K Broste, ${ }^{4}$ Robert P Frantz, ${ }^{5}$ John M Davis, ${ }^{3,6}$ Amit Ringel, ${ }^{1}$ Chirayath M Suchindran, ${ }^{7}$ \\ Joseph R Hibbeln 1
}

1 Section on Nutritional

Neurosciences, Laboratory of

Membrane Biochemistry and

Biophysics, National Institute

on Alcohol Abuse and

Alcoholism, National Institutes

of Health, Bethesda, MD, USA

2Department of Physical

Medicine and Rehabilitation,

Program on Integrative

Medicine, University of North

Carolina, Chapel Hill, NC, USA

${ }^{3}$ Department of Psychiatry,

University of North Carolina,

Chapel Hill, NC, USA

${ }^{4}$ Medtronic, Minneapolis, MN, USA

${ }^{5}$ Division of Cardiovascular

Diseases, Mayo Clinic,

Rochester, MN, USA

${ }^{6}$ Psychiatric Institute, University of Illinois at Chicago, Chicago,

IL, USA

7Department of Biostatistics, UNC Gillings School of Global Public Health, Chapel Hill, NC, USA

Correspondence to: CE Ramsden Chris.Ramsden@nih.gov

Additional material is published online only. To view please visit the journal online.

Cite this as: $B M J$ 2016;353:i1246 http://dx.doi.org/10.1136/bmj.i1246

Accepted: 19 February 2016

\section{ABSTRACT}

OBJECTIVE

To examine the traditional diet-heart hypothesis through recovery and analysis of previously unpublished data from the Minnesota Coronary Experiment (MCE) and to put findings in the context of existing diet-heart randomized controlled trials through a systematic review and meta-analysis.

DESIGN

The MCE (1968-73) is a double blind randomized controlled trial designed to test whether replacement of saturated fat with vegetable oil rich in linoleic acid reduces coronary heart disease and death by lowering serum cholesterol. Recovered MCE unpublished documents and raw data were analyzed according to hypotheses prespecified by original investigators. Further, a systematic review and meta-analyses of randomized controlled trials that lowered serum cholesterol by providing vegetable oil rich in linoleic acid in place of saturated fat without confounding by concomitant interventions was conducted.

\section{SETTING}

One nursing home and six state mental hospitals in Minnesota, United States.

\section{PARTICIPANTS}

Unpublished documents with completed analyses for the randomized cohort of 9423 women and men aged 20-97; longitudinal data on serum cholesterol for the 2355 participants exposed to the study diets for a year or more; 149 completed autopsy files.

INTERVENTIONS

Serum cholesterol lowering diet that replaced saturated fat with linoleic acid (from corn oil and corn

\section{WHAT IS ALREADY KNOWN ON THIS TOPIC}

The traditional diet-heart hypothesis predicts that replacing saturated fat with vegetable oils rich in linoleic acid will reduce cardiovascular deaths by lowering serum cholesterol

This paradigm has never been causally demonstrated in a randomized controlled trial and thus has remained uncertain for over 50 years

Key findings from landmark randomized controlled trials including the Sydney Diet Heart Study and the Minnesota Coronary Experiment (MCE) were not fully published

\section{WHAT THIS STUDY ADDS}

Though the MCE intervention lowered serum cholesterol, this did not translate to improved survival

Paradoxically, MCE participants who had greater reductions in serum cholesterol had a higher, rather than lower, risk of death

Results of a systematic review and meta-analysis of randomized controlled trials do not provide support for the traditional diet heart hypothesis

oil polyunsaturated margarine). Control diet was high in saturated fat from animal fats, common margarines, and shortenings.

\section{MAIN OUTCOME MEASURES}

Death from all causes; association between changes in serum cholesterol and death; and coronary atherosclerosis and myocardial infarcts detected at autopsy.

\section{RESULTS}

The intervention group had significant reduction in serum cholesterol compared with controls (mean change from baseline $-13.8 \% v-1.0 \% ; \mathrm{P}<0.001)$. Kaplan Meier graphs showed no mortality benefit for the intervention group in the full randomized cohort or for any prespecified subgroup. There was a $22 \%$ higher risk of death for each $30 \mathrm{mg} / \mathrm{dL}(0.78 \mathrm{mmol} / \mathrm{L})$ reduction in serum cholesterol in covariate adjusted Cox regression models (hazard ratio 1.22, 95\% confidence interval 1.14 to $1.32 ; \mathrm{P}<0.001)$. There was no evidence of benefit in the intervention group for coronary atherosclerosis or myocardial infarcts. Systematic review identified five randomized controlled trials for inclusion ( $\mathrm{n}=10 \mathrm{808})$. In metaanalyses, these cholesterol lowering interventions showed no evidence of benefit on mortality from coronary heart disease $(1.13,0.83$ to 1.54$)$ or all cause mortality (1.07, 0.90 to 1.27$)$.

\section{CONCLUSIONS}

Available evidence from randomized controlled trials shows that replacement of saturated fat in the diet with linoleic acid effectively lowers serum cholesterol but does not support the hypothesis that this translates to a lower risk of death from coronary heart disease or all causes. Findings from the Minnesota Coronary Experiment add to growing evidence that incomplete publication has contributed to overestimation of the benefits of replacing saturated fat with vegetable oils rich in linoleic acid.

\section{Introduction}

The traditional diet-heart hypothesis ${ }^{12}$ predicts that the serum cholesterol lowering effects of replacing saturated fat with vegetable oil rich in linoleic acid will diminish deposition of cholesterol in the arterial wall, ${ }^{34}$ slow progression of atherosclerosis, ${ }^{5}$ reduce coronary heart disease events, and improve survival. ${ }^{67}$ This dietheart paradigm is supported by evidence from randomized controlled trials showing that replacement of saturated fat with linoleic acid lowers serum total cholesterol and low density lipoprotein ${ }^{8-12}$ and by observational evidence linking serum cholesterol to coronary heart disease events and deaths (fig 1). ${ }^{13}$ Despite these 


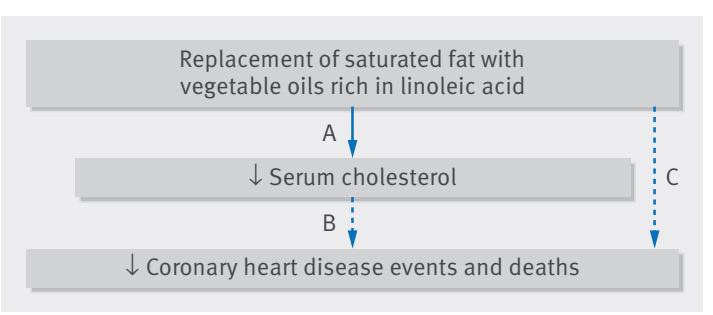

Fig 1 | Traditional diet-heart hypothesis. Solid line indicates that causal relation has been established by randomized controlled trials (A); dashed lines indicate that no causal relation has been established ( $B$ and $C$ ). $A=$ randomized controlled trials show that replacement of saturated fat with vegetable oils rich in linoleic acid lowers serum total cholesterol and LDL; $B=$ intermediate endpoints related to serum cholesterol are robustly associated with risk of coronary heart disease events and deaths in observational studies; $\mathrm{C}=$ randomized controlled trials have tested whether replacement of saturated fat with linoleic acid reduces coronary heart disease events or deaths; none have shown significant benefit

compelling relations, no randomized controlled trial has shown that replacement of saturated fat with linoleic acid significantly reduces coronary heart disease events or deaths (fig 1).

\section{Recovery of unpublished data could shift the evidence base}

Only a handful of randomized controlled trials have ever causally tested the traditional diet-heart hypothesis. The results for two of these trials were not fully reported. Our recovery and 2013 publication of previously unpublished data from the Sydney Diet Heart Study (SDHS, 1966-73) belatedly showed that replacement of saturated fat with vegetable oil rich in linoleic acid significantly increased the risks of death from coronary heart disease and all causes, despite lowering serum cholesterol. ${ }^{14}$ Our recovery of unpublished documents and raw data from another dietheart trial, the Minnesota Coronary Experiment, provided us with an opportunity to further evaluate this issue.

\section{Minnesota Coronary Experiment}

The Minnesota Coronary Experiment (MCE), a randomized controlled trial conducted in 1968-73, was the largest $(n=9570)$ and perhaps the most rigorously executed dietary trial of cholesterol lowering by replacement of saturated fat with vegetable oil rich in linoleic acid. The MCE is the only such randomized controlled trial to complete postmortem assessment of coronary, aortic, and cerebrovascular atherosclerosis grade and infarct status and the only one to test the clinical effects of increasing linoleic acid in large prespecified subgroups of women and older adults.

Despite the potential importance of this trial, critical analyses prespecified by the original MCE investigators have not been previously published or considered part of the evidence base (table 1). For example, we do not yet know the effects of the serum cholesterol lowering intervention in prespecified subgroups; the association between longitudinal changes in serum cholesterol and risk of death; or the effects of the intervention on any autopsy endpoints.

We recovered raw MCE data, including previously unpublished records of serum cholesterol and autopsy reports (table 1 and fig 2), and an extensive collection of study documents, including a 1981 master's thesis by S K Broste. The Broste thesis, which includes detailed survival analyses for the full randomized cohort and for

\begin{tabular}{|c|c|c|}
\hline Question and study population(s) & $\begin{array}{l}\text { Prior publication in peer } \\
\text { reviewed manuscript }{ }^{15}\end{array}$ & $\begin{array}{l}\text { Analyses of recovered raw } \\
\text { data in present manuscript }\end{array}$ \\
\hline \multicolumn{3}{|l|}{ Did MCE intervention lower serum cholesterol? } \\
\hline$\geq 1$ year cohort* & Published & Included \\
\hline Prespecified subgroups & - & Included \\
\hline \multicolumn{3}{|l|}{ Did MCE intervention reduce risk of death? } \\
\hline Full cohort & Published & Thesist \\
\hline Men and women $\neq$ & Published & Thesist \\
\hline$\geq 65 \S$ and $<65$ years & - & Thesist \\
\hline Primary and secondary prevention & - & $--^{\star \star}$ \\
\hline \multicolumn{3}{|l|}{ Was change in serum cholesterol related to risk of death? } \\
\hline$\geq 1$ year cohort* & - & Included \\
\hline Men and women $\neq$ & - & Included \\
\hline Age $\geq 65 \S$ and $<65$ & - & Included \\
\hline Primary and secondary prevention§ & - & $-\star \star \star$ \\
\hline \multicolumn{3}{|l|}{ Autopsy cohort } \\
\hline Did intervention reduce progression of aortic or coronary atherosclerosis? & - & Includedtt \\
\hline Did intervention reduce autopsy confirmed myocardial infarction? & - & Includedtt \\
\hline Did intervention reduce progression of atherosclerosis in circle of Willis (brain) or risk of stroke? & - & $-{ }^{\star \star *}$ \\
\hline \multicolumn{3}{|c|}{$\begin{array}{l}\text { *Serum cholesterol was measured only in subset of randomized participants with exposure to study diets for one year or longer. } \\
\text { †Raw data were not recovered; Kaplan Meier life tables included from } 1981 \text { Broste thesis. } \\
\text { fMCE is only randomized controlled trial to test whether replacement of saturated fat with linoleic acid rich vegetable oil reduced coronary heart disease or death in women. } \\
\text { \$MCE is larger of only two randomized controlled trials to test whether replacement of saturated fat with linoleic acid rich vegetable oil reduced coronary heart disease or death in older adults. } \\
\text { १392 MCE participants had electrocardiographic evidence of current or prior myocardial infarction (pathological Q wave) at randomization. } \\
\text { **Raw data not recovered. } \\
\text { ††Data recovered on } 149 / 295 \text { completed autopsies. }\end{array}$} \\
\hline
\end{tabular}




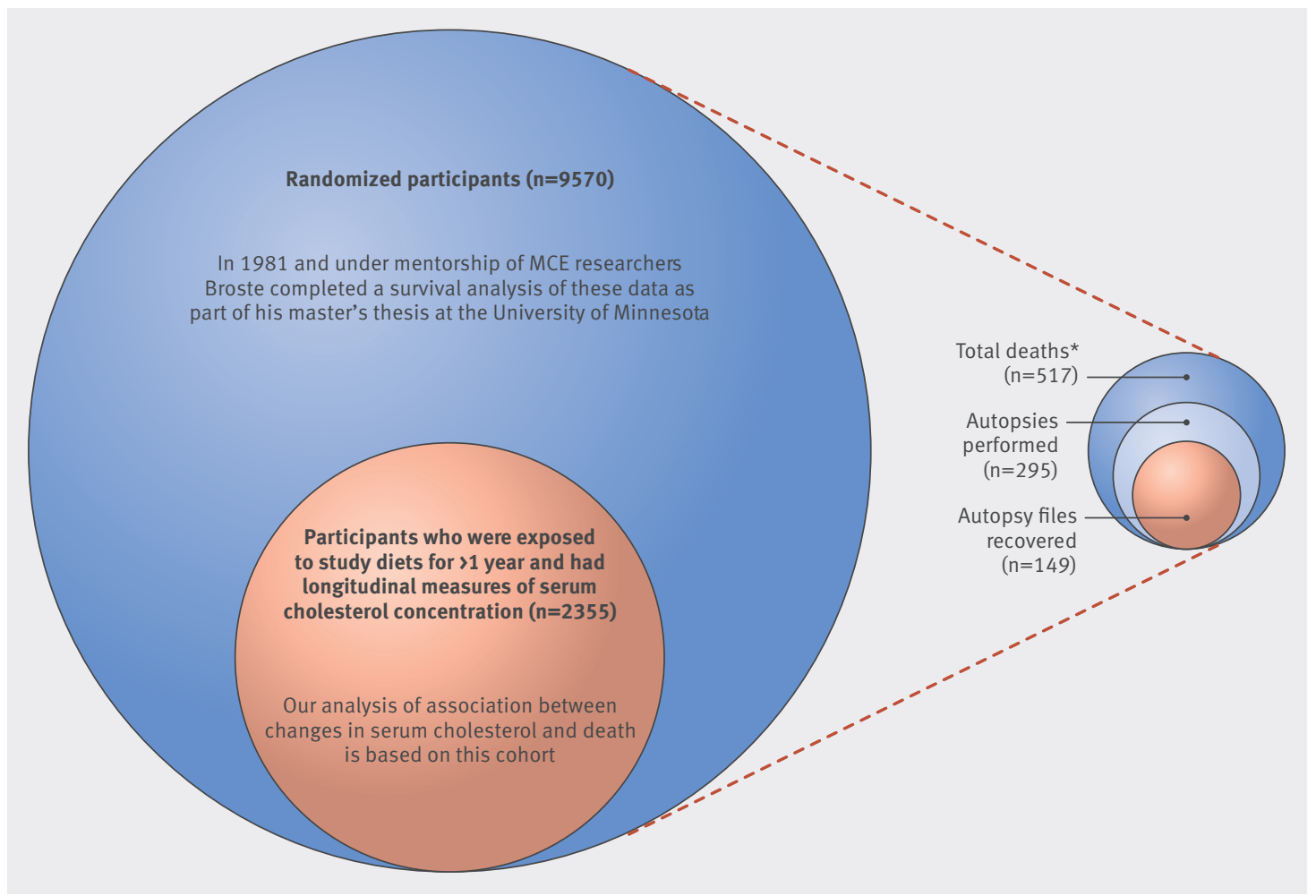

Fig 2 MCE total population and recovered cohorts (517 total deaths reported in 1989 publication by Frantz and colleagues ${ }^{15}$ )

the prespecified age and sex subgroups, has never previously been cited or considered part of the scientific evidence base (http://www.psych.uic.edu/download/ Broste_thesis_1981.pdf).

We analyzed recovered raw data according to hypotheses prespecified by the original study investigators (table 1). Further, to put these MCE findings into context, we conducted a systematic review and metaanalysis of all available randomized controlled trials that specifically tested whether replacement of saturated fat with linoleic acid rich oils reduces risk of death from coronary heart disease and all cause mortality.

\section{Methods}

Study design and participants

The MCE was a double blinded, parallel group, randomized controlled dietary intervention trial, designed to evaluate the effects of increasing n-6 linoleic acid from corn oil in place of saturated fat for primary and secondary prevention of cardiovascular events and deaths, and for reducing the degree of coronary, aortic, and cerebrovascular atherosclerosis, and the number of myocardial infarcts and strokes detected at autopsy. It was conducted from 1968 to 1973 in the state of Minnesota, United States. Eligible participants were men and women aged $\geq 20$ admitted to either the Oak Terrace Nursing Home or one of six state mental hospitals (Anoka, Fergus Falls, Hastings, Moose Lake, Saint Peter, Willmar). The experiment lasted from 41 to 56 months, depending on the hospital. The experiment was funded by the US Public Health Service and National Heart Institute through the R01 mechanism
(HE 09686), with Ivan Frantz as principal investigator and Ancel Keys as co-principal investigator.

\section{Ethical considerations for design and} implementation of MCE (1968-73)

The MCE was approved by the clinical research committee of the University of Minnesota and by each of the seven collaborating hospitals. ${ }^{15}$ No consent forms were required because the study diets were considered to be acceptable as house diets and the testing was considered to contribute to better patient care. ${ }^{15}$ Prior to beginning the diet phase, the project was explained and sample foods were served. Residents were given the opportunity to decline participation. Non-participants were served the control diet and did not provide blood samples or undergo electrocardiography. We were not able to recover a detailed description of the MCE autopsy consent procedure. According to the 1989 publication, $42.9 \%$ (corresponding to 222 of the 517 reported deaths) of those who died did not undergo autopsy, which was almost always because of "refusal by relatives or inability to contact relatives." 15

Patient involvement in present analysis of recovered data from MCE (2013-15)

No patients were involved in setting the research question or the outcome measures, nor were they involved in developing plans for design or implementation of the study. No patients were asked to advise on interpretation or writing up of results. There are no plans to disseminate the results of the research to study participants or the relevant patient community. 


\section{MCE data recovery and validation}

We obtained approval from the NIH Office of Human Research Protection (OHSRP \#5743) and collaboration from Robert Frantz to recover, analyze, and interpret de-identified MCE data and study related materials stored on two 9-track magnetic tapes (see appendix fig A) and an extensive collection of paper documents, including numerous green-bar paper files, paper autopsy folders, original and supplementary grant proposals, data collection forms, and FORTAN coding sheets. Part 1 of the appendix describes the methods used to recover data, convert into a useable format, verify accuracy, and merge into a master file. For further validation, we compared each of the recovered MCE datasets with each other and with data reported in the 1989 study publication, ${ }^{15}$ the 1981 master's thesis (Broste S K, Lifetable analysis of the Minnesota Coronary Survey http://www.psych.uic.edu/ download/Broste_thesis_1981.pdf), ${ }^{7}$ and 1975 conference proceedings, ${ }^{16-18}$ as well as numerous other recovered MCE documents and data sources as described in part 1 of the appendix. Previous mentions of this study, including the recovered documents, refer to the "Minnesota Coronary Survey (MCS)" because prior to randomization there was an observational phase that lasted almost three years. We call it Minnesota Coronary Experiment (MCE) to emphasize that we are using the experimental, randomized controlled trial phase of the MCS.

\section{Overview of original MCE objectives}

According to the MCE R01 grant proposal entitled "Effect of a Dietary Change on Human Cardiovascular Disease," the project objective was to "provide evidence concerning the possibility of reducing the incidence of clinical manifestations of atherosclerosis by dietary modification." 6 The MCE team planned to test whether the dietary intervention reduced coronary heart disease events and deaths in the total population and in prespecified subgroups of women, men, ages $\geq 65$ and $<65$, and participants with and without established coronary heart disease (primary and secondary prevention), with a special emphasis on participants exposed to study diets for a year or longer. ${ }^{619-21}$

Extensive postmortem data were collected to allow investigators to test the hypothesis that the serum cholesterol lowering diet would reduce coronary, aortic, and cerebrovascular atherosclerosis, as well as autopsy confirmed myocardial infarcts and strokes. ${ }^{21}$

There was no single prespecified primary outcome for this randomized controlled trial in any of the records we recovered, including the R01 grant application, detailed supplementary progress reports, and an extensive collection of FORTRAN coding sheets. In each of these documents, an emphasis was placed on total deaths, deaths from coronary heart disease, and non-fatal coronary heart disease events.

\section{MCE hypothesis and endpoints specified} in funded proposal

Power and sample size considerations

The recovered documents did not contain a traditional sample size calculation. This was likely because of the lack of a prespecified primary endpoint. We did recover multiple power calculations with different endpoints and assumptions, which provide ranges for adequate sample sizes. For example, based on epidemiological associations between serum cholesterol and coronary heart disease events in non-randomized cohorts, the MCE investigators applied the Cornfield equation $\left[\right.$ risk $\left.=\mathrm{k}(\text { serum cholesterol })^{\mathrm{n}}\right]$ to predict that between 2490 and 11645 participants would be required to "obtain a difference in 5 years significant at the $95 \%$ confidence level" with $\alpha=\beta=0.05 .{ }^{19}$ Based on the rate of strictly defined deaths from coronary heart disease observed during the MCE observational phase, and the elimination of all participants staying in the hospital less than a year, the estimated duration of the experiment required to assess the efficacy of the intervention was 3.6 years (with $\alpha=\beta=0.05$ ). ${ }^{20}$ These calculations were made to allow 95\% power; typical $\beta$ used in randomized controlled trials today is 0.20 , which allows for $80 \%$ power.

\section{Timeline/setting}

\section{Pre-randomization phase}

The randomized controlled trial phase was preceded by a 33 month pre-randomization observational phase (February 1966 to November 1968), during which the study team characterized the hospital populations, developed and refined procedures for diet delivery, baseline and follow-up visits, sick visits, blood collection, electrocardiograms, and postmortem examination, as well as the data collection and management plans.

\section{Randomized controlled trial phase}

The experimental dietary intervention phase, which was initiated over a 15 month period according to start dates of hospital specific diets, lasted for a maximum of 56 months. The start dates and duration of diet for each hospital are presented in table B in the appendix. Participants were followed up only while they were inpatients at the study hospitals. Participants who were admitted to a given hospital after its respective diet phase was underway completed baseline risk assessment, electrocardiographic testing, and serum collection before they started the study diets.

\section{Study diets}

\section{Pre-randomization hospital diet}

Prior to randomization, each hospital's food production program was covered by free provision of surplus USDA food commodities (common margarine, shortening, skim milk, flour, and rice) and Minnesota state funding. ${ }^{6}$ The average baseline hospital diet provided $18.5 \%$ and $3.8 \%$ of calories as saturated fatty acids and polyunsaturated fatty acids, respectively ${ }^{16}$ (fig 3). Based on the traditional distribution of polyunsaturated fatty acid species in US diets (about 90\% of which are linoleic acid), this baseline hospital diet provided about $3.4 \%$ of calories as linoleic acid.

\section{Serum cholesterol lowering diet}

The MCE experimental serum cholesterol lowering diet was derived from the "BC" diet of the institutional arm 

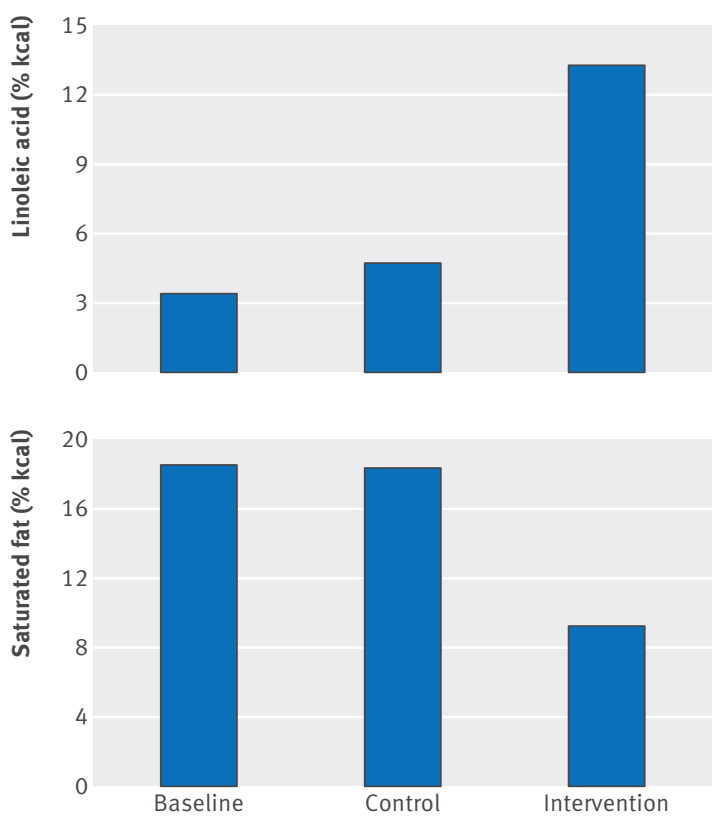

Fig 3 | Linoleic acid and saturated fat compositions of MCE control and intervention group diets. Values in figure are based on chemical analysis of study foods. ${ }^{16}$ Intervention group reduced saturated fat intake by about $50 \%$ and increased linoleic acid intake by $>280 \%$. Control group maintained high saturated fat intake but increased linoleic acid intake by about $38 \%$. Based on Keys equation, these diet changes are predicted to lower cholesterol in both groups (table 2)

of the National Diet-Heart Feasibility Study at Faribault Hospital. ${ }^{2615162022}$ Liquid corn oil was used in place of the usual hospital cooking fats (including hydrogenated oils) and was also added to numerous food items (for example, salad dressings, filled beef (lean ground beef with added oil), filled milk, and filled cheeses). Soft corn oil polyunsaturated margarine was used in place of butter. This intervention produced a mean reduction in dietary saturated fat by about 50\% (from $18.5 \%$ to $9.2 \%$ of calories) and increased linoleic acid intake by more than $280 \%$ (from about $3.4 \%$ to $13.2 \%$ of calories) ${ }^{1516}$ (fig 3 ). Hospital specific fatty acid compositions based on chemical analysis of a three week supply of study foods in 1971 are shown in part 1 of the appendix. There was substantial variability in study diets between hospitals, with saturated fat ranging from $8.0 \%$ to $12.3 \%$ and linoleic acid ranging from about $11.3 \%$ to $16.5 \%$ of calories. Saturated fat, however, was markedly reduced, and linoleic acid was markedly increased, in each hospital. Based on the average dietary changes, the Keys equation predicts that the MCE intervention diet would produce a marked reduction in serum cholesterol concentration (fig 3 and table 2).

\section{Control diet}

The control MCE diet was patterned after the "D" diet of the National Diet Heart Study. It was designed to appear similar to the experimental diet. Notably, free surplus USDA food commodities including common margarines and shortenings were key components of the control diet, making the daily per participant allocation from the state of Minnesota adequate to cover the full costs. ${ }^{21516}$ As common margarines and shortenings of this period were rich sources of industrially produced trans fatty acids, ${ }^{23-25}$ the control diet contained substantial quantities of trans fat. Compared with the prerandomization hospital diet, the control diet did not change saturated fat intake but did substantially increase linoleic acid intake (by about 38\%, from 3.4\% to $4.7 \%$ of calories). Based on this increase in dietary linoleic acid alone, the Keys equation predicts that the control diet would lower average serum cholesterol compared with baseline (fig 3 and table 2). This reduction, however, would be modest compared with the reduction in the intervention group.

\section{Randomization/masking Randomization}

The original hospital inpatient population was randomized according to a stratified randomization scheme with 512 cells on the basis of eight variables (age, sex, length of stay in the hospital, weight, blood pressure, diabetes, cigarette smoking, and electrocardiographic evidence of previous myocardial infarction). When new patients were admitted to a hospital after the diet start date, the stratified randomization scheme used four cells, according to age and sex..$^{15}$ We did not recover specific details on implementation of randomization and concealment of allocation.

\section{Masking}

Study participants, the principal investigator, other study physicians, nurses, nutritionists, assistants, laboratory technicians, pathologists, and all other study staff were masked to group assignment.

Study foods were designed to appear similar in both groups. Both diets were served in a single line. Each study participant received his or her group specific food

\begin{tabular}{|c|c|c|c|c|}
\hline & \multicolumn{2}{|c|}{ Observed dietary changes* } & \multicolumn{2}{|l|}{ Serum cholesterol \% changes } \\
\hline & LA (\% change) & SFA (\% change) & Predicted based on Keys equationt & Observed in MCE $(n=2355) \neq$ \\
\hline Control diet & 38 & -1 & $-1.1 \%$ & $-1.0 \%$ (SD 14.5\%), $P<0.001$ \\
\hline
\end{tabular}


tray based on a unique computer generated code number, which was designed to be incomprehensible to the participants but easily interpreted by the food servers. ${ }^{15}$ Twenty one labels were printed out for each participant each week. Labels remaining on the sheet were used to record missed meals, which were transferred to a "Port-o-Punch card" for later assessment of the correlation between adherence (defined as the percentage of meals received) and cholesterol response.

\section{Assessment of clinical outcomes and intermediate endpoints \\ Data management}

Fifteen MCE forms were devised for recording the data from the hospitals and laboratories (appendix 2). The data collected on these forms and the adherence data collected on the punch cards were transferred to magnetic tapes for later analysis.

\section{Baseline assessment and routine follow-up assessments}

On study entry and at six month intervals thereafter throughout both the pre-randomization and randomized controlled trial phases, a project technician carried out a brief evaluation of the participant's risk status, ${ }^{6}$ fasting serum was sealed under nitrogen and stored at $-20^{\circ} \mathrm{C}$, and an electrocardiogram was obtained.

\section{Intermediate endpoints: serum cholesterol and triglyceride assays}

Serum cholesterol and triglyceride assays were performed according to the standard protocol of the Lipid Research Clinics ${ }^{1526}$ in a laboratory standardized and monitored by the Center for Disease Control (Atlanta, GA).

\section{Serum cholesterol measurements in cohort receiving study diets for a year or more}

MCE investigators hypothesized that the clinical effects of lowering serum cholesterol would take substantial time to manifest and thus placed special emphasis on the subgroup of participants exposed to the study diets for a year or more. ${ }^{6151920}$ MCE investigators chose not to measure serum cholesterol in the whole sample of participants exposed to diets for less than a year because they "remained in the hospital for too short a time to contribute significantly to the results." 15 Based on recovered data, 2403 MCE participants were exposed to study diets for a year or more. We recovered longitudinal data on serum total cholesterol for 2355 (98\%) of these participants. In addition to pre-randomization measures, participants had an average of six follow-up measurements of serum cholesterol.

\section{Evaluation of clinical events and deaths}

MCE investigators categorized fatal and non-fatal events into 10 categories (table $\mathrm{C}$ in the appendix). They used a conservative approach to attribute the cause of death to coronary heart disease. The MCE team noted "reluctance to classify a death in this category unless objective evidence is at hand." Close attention was therefore directed to the overall death rate as "atherosclerosis may contribute to many deaths in which no actual fresh myocardial infarct or coronary occlusion has occurred.” ${ }^{20}$ We recovered data on all cause mortality but did not recover data on non-fatal events or deaths from coronary heart disease. Data on coronary heart disease deaths in relation to intermediate endpoints existed, however, and were reported in the 1981 Broste thesis.

Postmortem examination of heart, aorta, and brain The MCE grant proposal, supplements, and FORTRAN coding sheets ${ }^{619-21}$ included detailed plans for analysis of autopsy data, and a 9-track magnetic tape file with full autopsy data (MCE Tape \#380) (appendix part 1) was known to exist. To our knowledge, however, no autopsy results have ever been published or reported. According to the 1989 publication, 57.1\% (corresponding to 295 of the 517 reported deaths) of participants who died during the experimental dietary intervention phase underwent autopsy. Hearts, aortas, and brains were sent to the University of Minnesota for blinded grading by university pathologists. We recovered heart and aorta autopsy files for 149 out of these 295 completed autopsies. The remaining autopsy files remain unaccounted for.

\section{Degree of coronary atherosclerosis and number of myocardial infarctions}

The degree of coronary atherosclerosis and mapping of myocardial infarcts were evaluated by the multiple cross section technique as described by Spiekerman and colleagues. ${ }^{2021}$ At each of 16 coronary vessel sites, vessel narrowing was scored on a 4 point scale based on the percentage closure of the coronary lumen with $1,2,3$, and 4 signifying $<25 \%$, $25-50 \%, 50-75 \%$, and $100 \%$, respectively. These data were recorded on MCE form No 8 (appendix 2). Scores at each site were summed to calculate a total coronary atherosclerosis score (range 16-64). Subendocardial and transmembrane infarcts were identified after the ventricles were cut into transverse sections and recorded on MCE form No 9 (appendix 2).

\section{Degree of aortic atherosclerosis}

The degree of aortic atherosclerosis was graded from 1 to 7 according to the technique devised by the Committee on Grading of Lesions of the Council of Atherosclerosis of the American Heart Association ${ }^{20}$ and recorded on form No 10 (appendix 2). Each aorta was graded independently by two observers without knowledge of age, sex, diagnosis, hospital of origin, or diet group. An aortic atherosclerosis score variable was created by taking the average of the two measures for each individual.

\section{Data analysis}

Effect of cholesterol lowering intervention

We were unable to recover the complete MCE dataset including all randomized participants. Thus, we could not conduct a traditional randomized controlled trial analysis to determine the effect of randomization. We do, however, include the life table graphs from the 


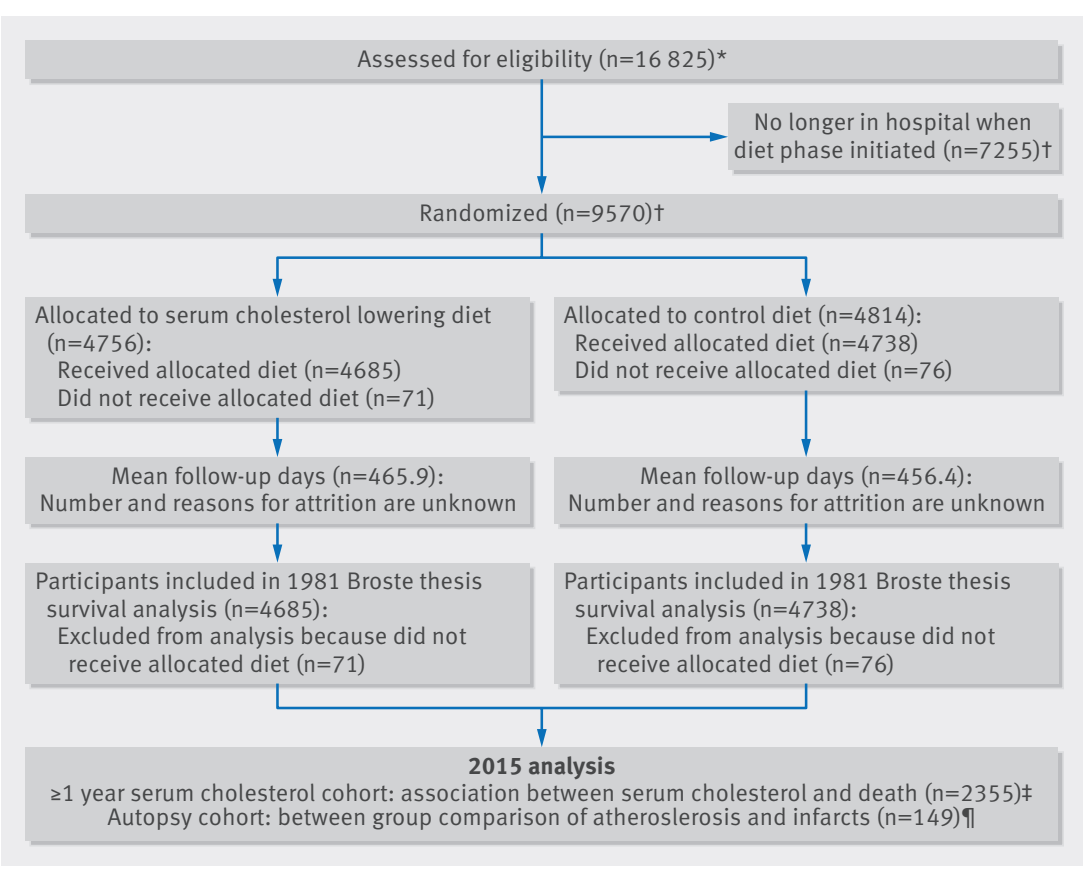

Fig 4 | MCE flow diagram. *16 825 participants completed MCE form No 011 (tape 4 data, appendix part 1); $†$ Broste thesis reports that 9570 participants were randomized and that 147 dropped out prior to diet exposure, 1975 abstract and 1989 manuscript reported that 9449 and 9057 participants were randomized, respectively; ¥longitudinal serum cholesterol data recovered for 2355/2403 randomized participants exposed to diet for one year or more (tape 2 data, appendix part 1); ๆlautopsy files with aortic and coronary atherosclerosis and infarct data recovered for 149/295 completed autopsies

1981 Broste thesis. A thorough explanation of methods and results can be found on pages 21-49 of the Broste thesis (http://www.psych.uic.edu/download/Broste thesis_1981.pdf). For context, we include a CONSORT flow diagram (fig 4).

\section{6 analysis}

Our analyses used longitudinal data for the 2355 participants who received the intervention diet for a year or more and were carried out in Stata version 13.1. To determine the effect of the dietary intervention on serum cholesterol, we analyzed group differences in serum cholesterol over time using a generalized estimating equation model with timexgroup interaction.

\section{Association between changes in serum cholesterol and risk of death}

First, we provide a crude visual representation of the association between cholesterol and death by graphing the distribution of change in total serum cholesterol concentration (using the average of measurements for each participant before and after randomization) along with the number and percentage of deaths followed by plots of age adjusted logistic regression models.

For our main analysis, we used Cox regressions for death as a function of continuous time varying serum cholesterol (up to 12 repeated measures on each participant) and adjusted for clustering within hospital. We used Schoenfeld residuals to test the proportional hazards assumption and martingale residuals to assess model fit. We present results from crude models, models adjusted for relevant variables including age, sex, blood pressure, BMI, and adherence to diet (percent of missed meals), and sensitivity analyses further adjusting for time varying changes in BMI and systolic blood pressure. All models were tested for effect modification by diet group. For simplicity, we have shown hazard ratios for each $30 \mathrm{mg} / \mathrm{dL}(0.78 \mathrm{mmol} / \mathrm{L})$ decrease in serum cholesterol.

\section{Atherosclerotic progression and number of myocardial infarcts confirmed at autopsy}

As we recovered only 149 of the original 295 autopsy files, our analysis of the effects of the serum cholesterol lowering diet on atherosclerotic progression and myocardial infarcts confirmed at autopsy should be considered provisional until the complete autopsy data are recovered. We calculated incidence rate ratios for the presence of at least one autopsy confirmed myocardial infarct according to diet group. We used linear regression to examine whether assignment to diet group or changes in serum cholesterol concentration were associated with coronary or aortic atherosclerosis. We used logistic regression to examine the association between serum cholesterol concentration and the presence of at least one autopsy confirmed myocardial infarct.

\section{Systematic review and meta-analysis of randomized} controlled trials replacing saturated fat with linoleic acid rich vegetable oils

A key component of dietary guidelines has long been to replace saturated fat with oils rich in linoleic acid (such as corn oil, sunflower oil, safflower oil, cottonseed oil, or soybean oil). This advice is based on the traditional diet-heart hypothesis prediction that replacement decreases coronary heart disease and all cause mortality. Thus, we followed PRISMA standards ${ }^{23}$ for reporting a systematic review and meta-analysis of randomized controlled trials that specifically tested whether replacement of saturated fat with vegetable oil rich in linoleic acid decreases mortality from coronary heart disease and all cause mortality. Included in the main analysis were all serum cholesterol lowering randomized controlled trials since 1950 with publication in English that randomly assigned individual participants; provided an intervention of vegetable oil rich in linoleic acid in place of saturated fat, compared with a usual care control diet; were not confounded by the addition of large quantities of n-3 EPA (eicosapentaenoic acid) and DHA (docosahexaenoic acid) or by other major concomitant interventions (such as complex changes in diet pattern) or unequal intensity of medical management (such as advice on smoking cessation or control of blood pressure); and reported deaths from coronary heart disease or all causes. Hence, we excluded randomized controlled trials that provided large quantities of n-3 EPA and DHA or provided advice only without provision of linoleic acid rich oils from the main analysis, and studies with only biochemical or intermediate endpoints. Sensitivity analyses included diet-heart randomized controlled trials that also pro- 


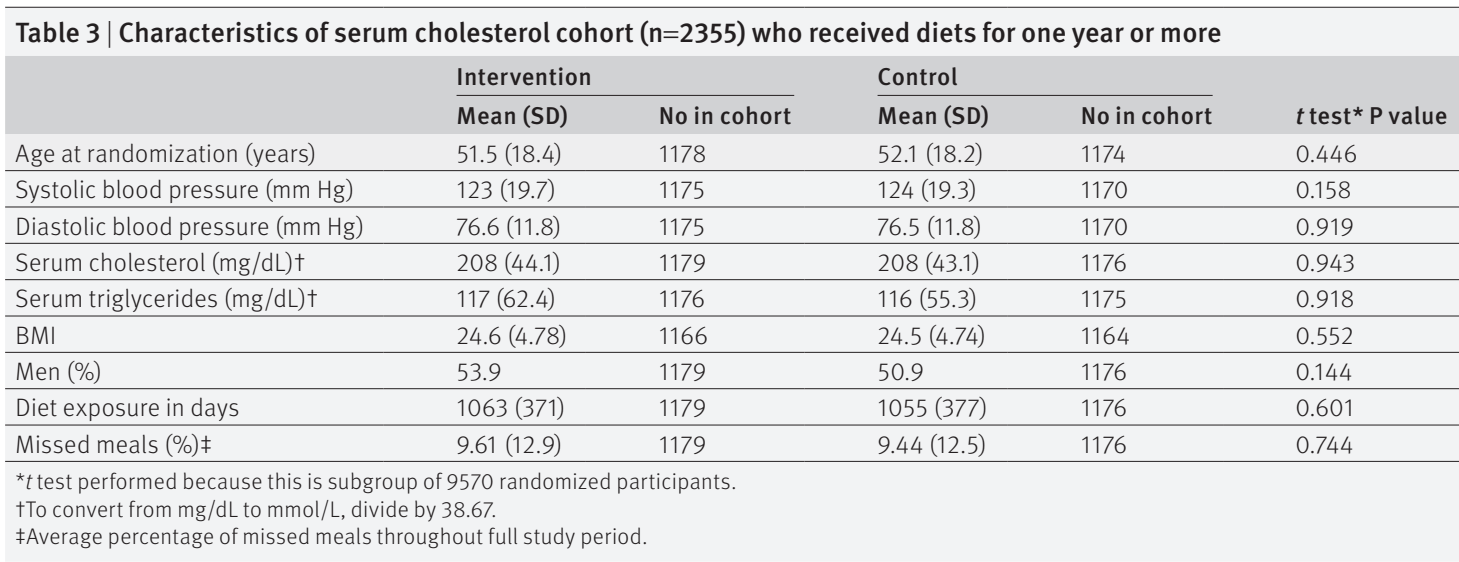

vided large quantities of n-3 EPA and DHA or provided advice only without provision of a linoleic acid rich study oil but otherwise met the inclusion and exclusion criteria for the main analysis.

A detailed description of the search strategy, study selection and data extraction, bias assessments, and statistical methods is included in part 2 of the web appendix.

\section{Results}

Baseline demographics and clinical characteristics Characteristics of the full MCE population are reported in the 1981 Broste thesis ( $\mathrm{n}=9423$; http://www.psych.uic. edu/download/Broste_thesis_1981.pdf). Table 3 shows characteristics of the serum cholesterol cohort $(n=2355)$ who received the diets for a year or more. The intervention and control groups were well balanced at baseline, with no detectable differences in any of the recovered variables. The age ranged from 20 to 97, with a mean age of 52 . Slightly more than half were women, and $25 \%$ were aged 65 or older. Average BMI was 24.5 and average serum cholesterol concentration was $208 \mathrm{mg} / \mathrm{dL}$ (to convert from mg/ $\mathrm{dL}$ to $\mathrm{mmol} / \mathrm{L}$, divide by 38.67 (for example, $208 \mathrm{mg} / \mathrm{dL} /$ $38.67=5.39 \mathrm{mmol} / \mathrm{L})$ ). Mean follow-up for participants in this cohort was 2.9 years (median 3.1 years).

Did the MCE intervention lower serum cholesterol? MCE investigators hypothesized that replacing saturated fat with vegetable oil rich in linoleic acid would lower serum cholesterol in a manner consistent with the Keys equation. ${ }^{27}$ As predicted, participants in the intervention group significantly lowered serum cholesterol compared with the control group $(\mathrm{P}<0.001)$ and compared with baseline (mean change $-31.2 \mathrm{mg} / \mathrm{dL}$, (SD $30.6 \mathrm{mg} / \mathrm{dL}$ ); -13.8\% (SD 13.0\%); $\mathrm{P}<0.001$ ) (table 2). Participants in the intervention group also reduced serum cholesterol in each of the prespecified subgroups of sex and age (all $\mathrm{P}<0.01$ ).

Among the intervention group, higher adherence (fewer missed meals) was associated with a more pronounced reduction in serum cholesterol $(\mathrm{P}<0.001)$. Participants who missed $\leq 2 \%$ of their meals $(n=390)$ achieved reductions in concentration of $-18.0 \%$, which is similar to that predicted by the Keys equation (table 2 and table E in appendix).
The control diet, which increased dietary linoleic acid by $38 \%$ but did not alter saturated fat, produced a modest but significant reduction in serum cholesterol compared with baseline $(-5 \mathrm{mg} / \mathrm{dL}$ (SD $30 \mathrm{mg} / \mathrm{dL}$ ); $-1.0 \%$ (SD 14.5\%); P<0.001) (fig 3 and table 2). Higher adherence to the control diet was also associated with greater reduction in serum cholesterol $(\mathrm{P}=0.004)$.

Did the MCE intervention reduce risk of death? To our knowledge, the most complete analysis comparing mortality in the intervention versus control group was reported in the Broste thesis. ${ }^{7}$ This included Kaplan Meier life table graphs for cumulative mortality for the full MCE cohort, and for each prespecified subgroup (fig 5). These life table graphs confirm that there was no mortality benefit in the full MCE cohort. Moreover, the life table for the cohort aged $\geq 65$ (fig 5 ) suggests the possibility of an increased risk of death for the intervention group compared with controls. In the thesis, Broste noted "the excess mortality in the diet group seems to have been confined primarily to patients 65 or older." 7 In the absence of the raw data, however, we cannot determine the statistical significance of this finding. A survival analysis that was presented in the 1989 manuscript ${ }^{15}$ also showed no mortality benefit in the full MCE population (subgroup analyses were not reported). Thus, collective data from the 1989 publication and 1981 Broste thesis provide no evidence for mortality benefit and suggest the possibility of increased risk of death in older adults.

Was the change in serum cholesterol related to risk of death?

The traditional diet heart hypothesis predicts that participants with greater reduction in serum cholesterol would have a lower risk of death (fig 1, line B). MCE participants with greater reduction in serum cholesterol, however, had a higher rather than a lower risk of death. Figure 6 provides a simplified visual representation of change in serum cholesterol and death in the intervention group, control group, and combined groups; table 4 provides more advanced statistical analyses, with hazard ratios for crude, adjusted, and sensitivity models. The average change in serum cholesterol in the intervention, control, and combined groups was -31 

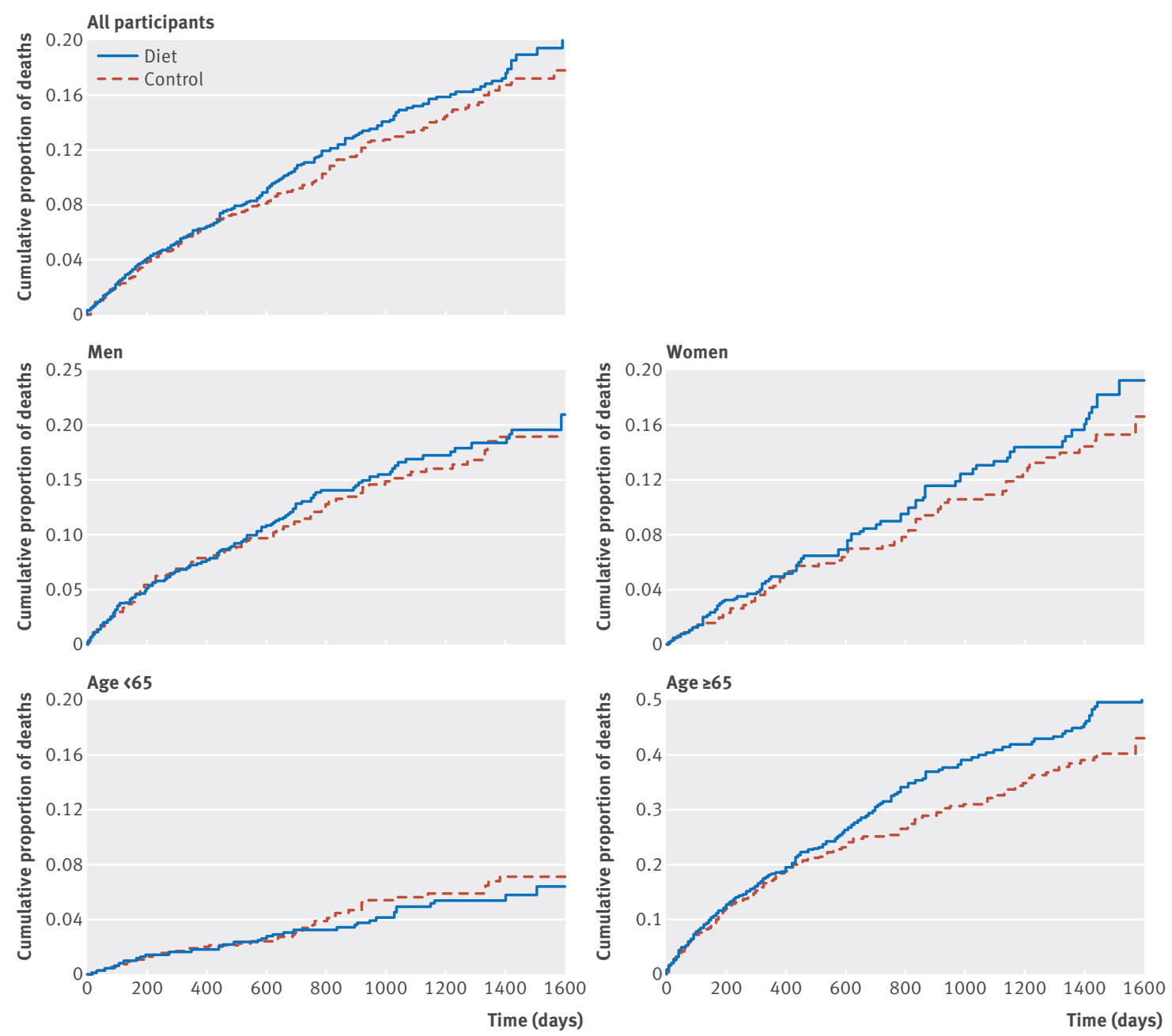

Fig 5 Risk of death from any cause by diet assignment in full MCE cohort and prespecified subgroups (Kaplan Meier life table graphs of cumulative mortality). Graphical depiction of cumulative mortality in full MCE cohort ( $n=9423)$ and prespecified subgroups in 1981 Broste thesis ${ }^{7}$ showed no indication of benefit and suggested possibility of unfavorable effects of serum cholesterol lowering intervention among participants aged $\geq 65$. Patient level data needed to repeat this analysis were not recovered

(SD 31), -5 (SD 30), and -18 (SD 33) mg/dL, respectively (fig 6, top row). The number, proportion, and probability of death increased as serum cholesterol decreased (fig 6, rows 2, 3, 4).

In survival analyses (table 4), there was a robust association between decreasing serum cholesterol and increased risk of death, and this association did not differ between the intervention and control group $(\mathrm{P}>0.16$ for all serum cholesterol $\times$ intervention interactions). Among both groups combined, a $30 \mathrm{mg} / \mathrm{dL}(0.78 \mathrm{mmol} / \mathrm{L})$ decrease in serum cholesterol was associated with $22 \%$ higher risk of death from any cause (hazard ratio 1.22, 95\% confidence interval 1.14 to 1.32 ) based on a Cox model adjusted for baseline serum cholesterol, age, sex, adherence to diet, BMI, and systolic blood pressure.

The higher risk of death associated with decreased serum cholesterol seems to be driven by the subgroup aged $\geq 65$. Among participants who were older than 65 at baseline, a $30 \mathrm{mg} / \mathrm{dL}$ decrease in serum cholesterol was associated with 35\% higher risk of death (hazard ratio 1.35 , 95\% confidence interval 1.18 to 1.54 ), whereas among people aged under 65 at baseline there was no relation between the change in serum cholesterol and death (1.01, 0.88 to 1.16$)$.

\section{Sensitivity analysis to account for frailty}

To explore the possibility that frailty (which is associated with both low cholesterol and death ${ }^{2829}$ ) could confound these results, we did a sensitivity analysis adjusting our Cox models (table 4) for two known markers of frailty (changes in body weight and changes in systolic blood pressure). ${ }^{28-30}$ These adjustments did not materially change the effect estimates, which remained significant in both groups.

This finding that greater lowering of serum cholesterol was associated with a higher rather than a lower risk of death in the MCE does not provide support for the traditional diet-heart hypothesis.

\section{Provisional autopsy findings}

Characteristics of the partially recovered autopsy cohort are shown in table $\mathrm{F}$ in appendix; the intervention and 

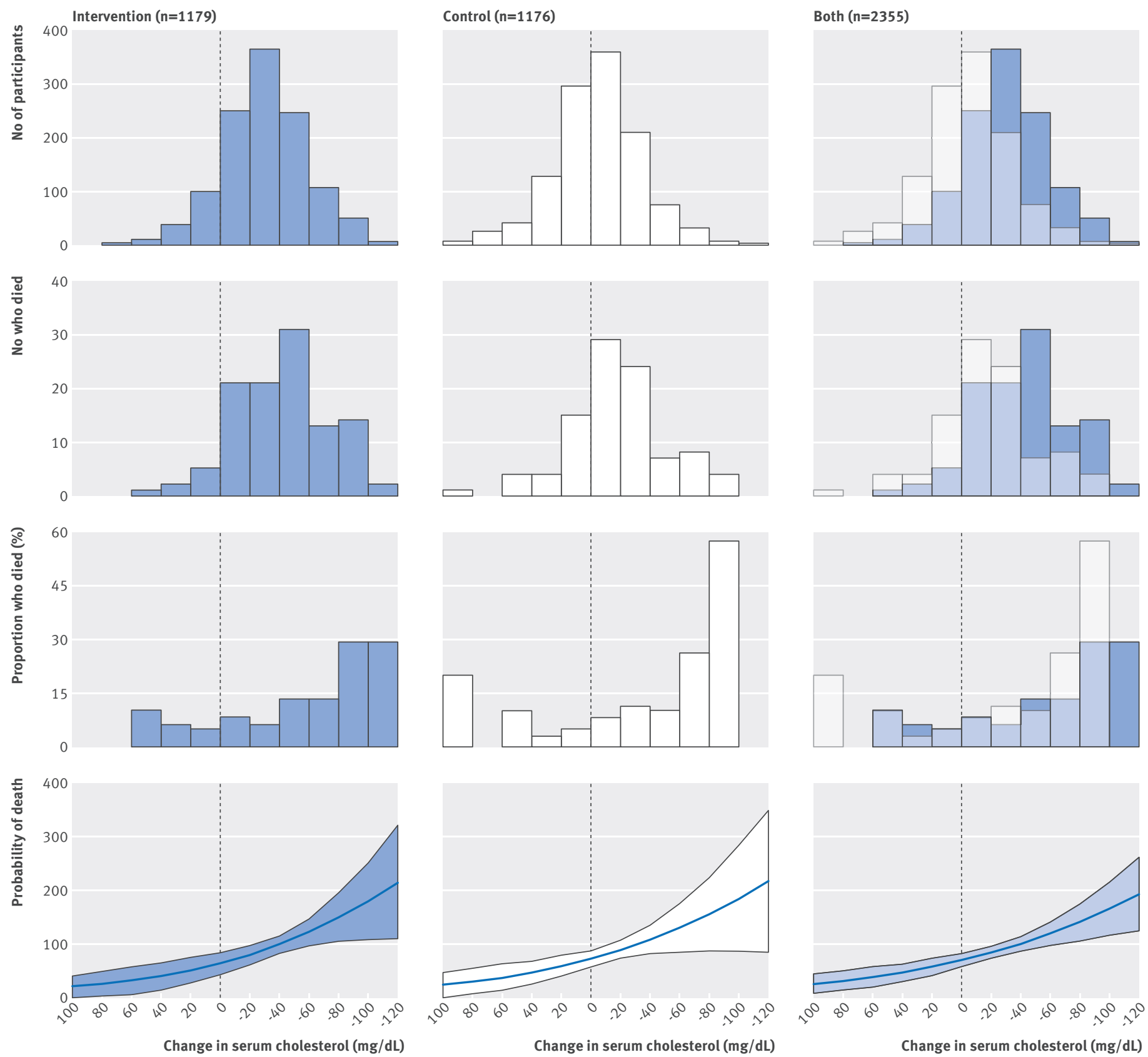

Fig 6 | Death from any cause and change in serum cholesterol in cohort that received diets for one year or more $(n=2355)$. Panels indicate relations between change in serum cholesterol and number of participants, number of deaths, percent of deaths, and probability of death among intervention, control, and combined groups. Change in serum cholesterol calculated with average of measurements before and after randomization for each individual. Last row represents logistic model for death as function of average change in cholesterol, adjusted for age at baseline. Likelihood ratio test used to test effect modification by diet group $(\mathrm{P}=0.67)$

control groups were well balanced at baseline, with no evident differences for any demographic, clinical, or laboratory variables. The mean age was $69.5,36 \%$ were women, and the median follow-up was 298 days (316 days for intervention group, 217 days for control group). Baseline serum cholesterol concentration was $210 \mathrm{mg} / \mathrm{dL}$. The mean change in serum cholesterol during follow-up was $-17.9 \%$ in the intervention group and $-1.3 \%$ in the control group.

MCE investigators hypothesized that participants in the intervention group would have fewer myocardial infarcts confirmed by autopsy and less advanced atherosclerosis. In this autopsy cohort, however, 41\% (31/76) of participants in the intervention group had at least one myocardial infarct, whereas only 22\% (16/73) of participants in the control group did (incidence rate ratio 1.90, 95\% confidence interval 1.01 to 3.72; $\mathrm{P}=0.035$ ). Also, participants in the intervention group did not have less coronary atherosclerosis or aortic atherosclerosis (table 5). These findings should be interpreted with caution because of partial recovery of autopsy files. There was no association between serum 


\begin{tabular}{|c|c|c|c|}
\hline & Intervention & Control & Both groups \\
\hline No in group & 1179 & 1176 & 2355 \\
\hline \multicolumn{4}{|c|}{ HR (95\% Cl), P value: } \\
\hline Sensitivity§ & 1.20 (1.03 to 1.41), 0.023 & 1.32 (1.11 to 1.57), 0.002 & 1.24 (1.15 to 1.33$),<0.001$ \\
\hline \multicolumn{4}{|c|}{ Participants aged $<65$} \\
\hline No in group & 886 & 874 & 1760 \\
\hline \multicolumn{4}{|c|}{ HR (95\% Cl), P value: } \\
\hline Crudet & 1.02 (0.60 to 1.73), 0.936 & 1.17 (0.82 to 1.69), 0.389 & 1.07 (0.93 to 1.23), 0.368 \\
\hline No in group & 293 & 302 & 595 \\
\hline \multicolumn{4}{|c|}{ HR (95\% Cl), P value: } \\
\hline Crudet & 1.55 (1.39 to 1.71$),<0.001$ & 1.53 (1.24 to 1.88$),<0.001$ & $1.50(1.31$ to 1.71$),<0.001$ \\
\hline Adjusted $\neq$ & $1.42(1.22$ to 1.67$),<0.001$ & 1.39 (1.10 to 1.76$), 0.006$ & 1.35 (1.18 to 1.54$),<0.001$ \\
\hline Sensitivity§ & 1.36 (1.17 to 1.58$),<0.001$ & 1.43 (1.14 to 1.78$), 0.002$ & 1.35 (1.19 to 1.53$),<0.001$ \\
\hline \multicolumn{4}{|c|}{$\begin{array}{l}\text { *Cox regressions for death as function of time varying serum cholesterol concentration. All estimates represent hazard ratio for a serum cholesterol } \\
\text { decrease of } 30 \mathrm{mg} / \mathrm{dL}(0.78 \mathrm{mmol} / \mathrm{L}) \text {. All models account for clustering within hospital. } \\
\text { †Model adjusted for baseline serum cholesterol concentration. } \\
\text { †Adjusted for baseline serum cholesterol concentration, age, BMI, sex, adherence to diet, and systolic blood pressure (SBP). } \\
\text { §Further adjusted for time varying percentage change from baseline for BMI and SBP. }\end{array}$} \\
\hline
\end{tabular}

\begin{tabular}{|c|c|c|c|c|c|c|c|}
\hline & \multicolumn{2}{|l|}{ Intervention } & \multicolumn{2}{|l|}{ Control } & \multicolumn{3}{|c|}{ Intervention $v$ control } \\
\hline & No in group & Mean (SD) & No in group & Mean (SD) & No in group & Coef $(95 \% \mathrm{Cl}) \dagger$ & P value \\
\hline Aortic atherosclerosis score & 72 & $4.78(1.20)$ & 69 & $4.54(1.21)$ & 141 & $0.25(-0.15$ to 0.65$)$ & 0.223 \\
\hline
\end{tabular}

cholesterol and myocardial infarcts, coronary atherosclerosis, or aortic atherosclerosis in covariate adjusted models (table $\mathrm{G}$ in appendix).

Does the available body of evidence from randomized controlled trials support the traditional diet heart hypothesis?

The complete systematic review and meta-analysis following PRISMA guidelines is presented in appendix part 2. Briefly, out of 1270 screened records we identified only five randomized controlled trials that provided vegetable oil(s) rich in linoleic acid in place of saturated fat and were not confounded by unequal application of concomitant interventions. These five trials included 10808 participants, 324 deaths attributed to coronary heart disease, and 1001 deaths from all causes (table $\mathrm{K}$ and $\mathrm{L}$ in appendix). The mean change in serum cholesterol concentration in the course of the randomized controlled trials ranged from $7.8 \%$ to $13.8 \%$ lower in the intervention versus control groups. In meta-analyses of these five trials, there was no evidence of benefit on mortality from coronary heart disease (hazard ratio 1.13, 95\% confidence interval 0.83 to 1.54 ) (fig 7 ) or all cause mortality (1.07, 0.90 to 1.27) (fig D and E in appendix). In a sensitivity analysis, inclusion of three additional diet-heart trials that provided advice only and/or were confounded by the provision of large quantities of $n-3$
EPA+DHA did not appreciably alter results for mortality from coronary heart disease $(1.00,0.81$ to 1.24$)$ (fig 7 ) or all cause mortality (1.00, 0.87 to 1.15 ) (figs $\mathrm{H}$ and I in appendix). In sensitivity analyses that included non-fatal endpoints, there was no indication of benefit from the replacement of saturated fat with vegetable oils rich in linoleic acid, with either a composite outcome of myocardial infarcts plus death from coronary heart disease or non-fatal myocardial infarcts alone (fig K and L in appendix). Thus, although limited, available evidence from randomized controlled trials provides no indication of benefit on coronary heart disease or all cause mortality from replacing saturated fat with linoleic acid rich vegetable oils.

\section{Discussion}

The traditional diet-heart hypothesis predicts that replacing saturated fat with vegetable oil rich in linoleic acid will reduce coronary heart disease events and deaths by lowering serum cholesterol. Many studies have yielded results consistent with pieces of this hypothesis. The clinical benefits of these serum cholesterol lowering diets, however, have never been causally demonstrated in a randomized controlled trial and thus remain uncertain. We have recovered previously unpublished data from two landmark trials that were designed to provide causal evidence to support the diet-heart hypothesis. 


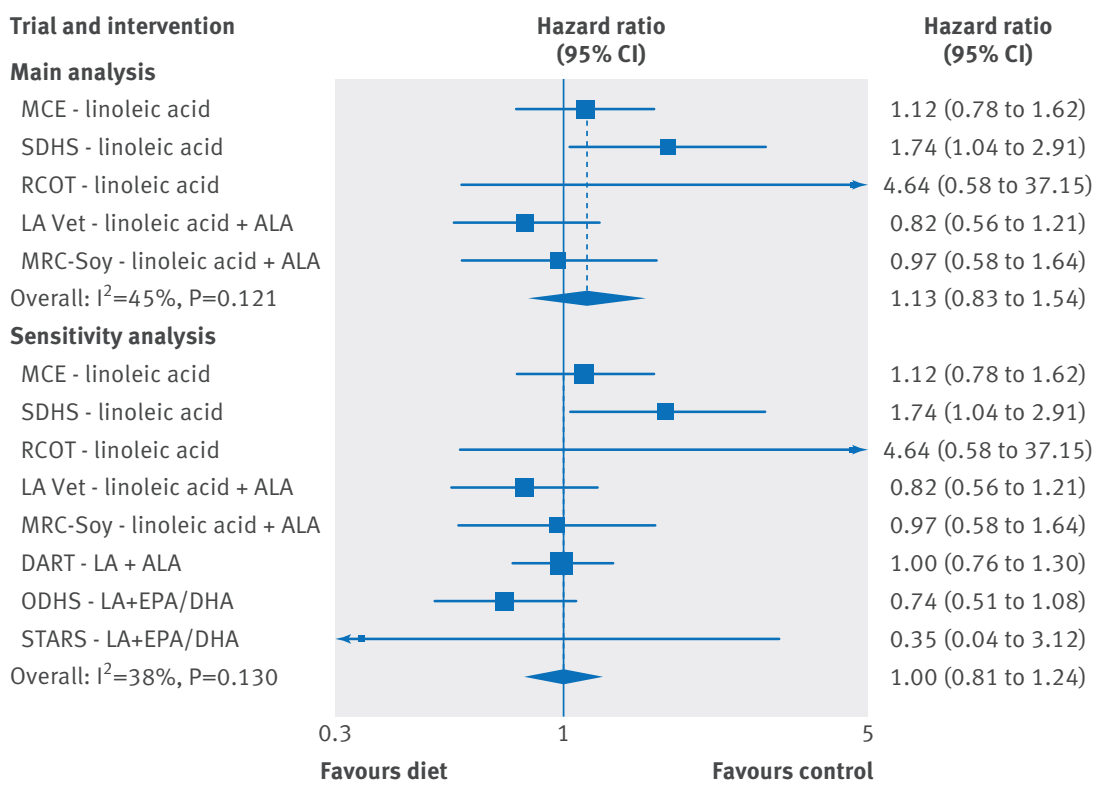

Fig 7 | Meta-analysis for mortality from coronary heart disease in trials testing replacement of saturated fat with vegetable oils rich in linoleic acid. Main analysis: trials provided replacement foods (vegetable oils) and were not confounded by any concomitant interventions. Sensitivity analysis: includes trials that provided advice only and/or were confounded by addition of n-3 EPA and DHA. Risk ratios were used as estimates of hazard ratios in MCE, RCOT, LA Vet, and MRC-Soy. MCE=Minnesota Coronary Experiment; SDHS=Sydney Diet Heart Study; RCOT=Rose Corn Oil Trial; LA Vet=Los Angeles Veterans Trial; MRC-Soy=Medical Research Council Soy Oil Trial; DART=Diet and Re-infarction Trial; ODHS=0slo Diet Heart Study; STARS=St. Thomas Atherosclerosis Regression Study; $\mathrm{LA}=$ linoleic acid; $\mathrm{SFA}=$ saturated fat; $\mathrm{ALA}=\alpha$ linolenic acid; $\mathrm{EPA}=$ eicosapentaenoate; $\mathrm{DHA}=$ docosahexaenoate (for example, healthy consumer bias), however, can sometimes distort, ${ }^{31-33}$ or even reverse, ${ }^{34-36}$ true associations. Self reported intake of foods that are often high in linoleic acid was associated with a lower risk of coronary heart disease in two large prospective observational cohorts of US health professionals ${ }^{3738}$ and in one pooled analysis of several cohorts. ${ }^{39}$ Other large prospective observational studies ${ }^{40}$ and another pooled analysis, ${ }^{41}$ however, found no association. Similar inconsistent associations between circulating linoleic acid and risk of coronary heart disease have been reported, ${ }^{4243}$ with pooled analysis showing no association..$^{41}$ Ecological associations between linoleic acid intake and coronary heart disease have also been cited to support the dietheart hypothesis. ${ }^{44}$ These associations, however, are subject to important confounders and are wholly dependent on the timeframe selected (fig B, appendix). Thus, the conclusions that can be drawn from non-randomized studies on this topic are limited. Together with the lack of support from randomized controlled trials (after recovery of data from the Sydney Diet Heart Study and MCE), the totality of evidence no longer provides support for the traditional diet-heart hypothesis.

\section{Why didn't lowering serum cholesterol translate to} clinical improvement in diet-heart randomized controlled trials?

A plausible explanation for the seemingly paradoxical results of the Sydney Diet Heart Study and MCE is that vegetable oil rich in linoleic acid was the agent used to lower serum cholesterol. As the major vehicle for delivery of cholesterol to vascular tissues, low density lipoprotein is often considered a causal mediator of coronary heart disease. ${ }^{45}$ As replacing saturated fat with linoleic acid specifically decreases low density lipoprotein (without affecting high density lipoprotein ${ }^{8}$ ) it is tempting to assume that such dietary changes will automatically translate to reduction in risk of coronary heart disease. Critically, however, consumption of vegetable oils rich in linoleic acid produces a wide range of biochemical consequences, including qualitative changes in lipoprotein particle oxidation that could plausibly increase risk of coronary heart disease. ${ }^{14} 46$ Hence the clinical effects of replacing saturated fat with vegetable oils could reflect the net impact of decreasing low density lipoprotein while increasing its susceptibility to oxidation. Moreover, low density lipoprotein concentrations are influenced by many factors, such as delivery of low density lipoprotein to blood vessels and other tissues, as well as hepatic clearance of native and oxidized low density lipoprotein particles. ${ }^{47-50}$ Therefore, a decrease in low density lipoprotein can represent widely different biochemical phenomena. This broader understanding could help to explain why some agents that decrease low density lipoprotein have been shown to reduce the risk of coronary heart disease, ${ }^{51} 52$ while others have no clear effect, ${ }^{53}$ and still others might actually increase risk. ${ }^{545}$ The collective data from diet-heart randomized controlled trials suggests that lowering serum cholesterol by replacing saturated fat with vegetable oils rich in linoleic acid has no clear 


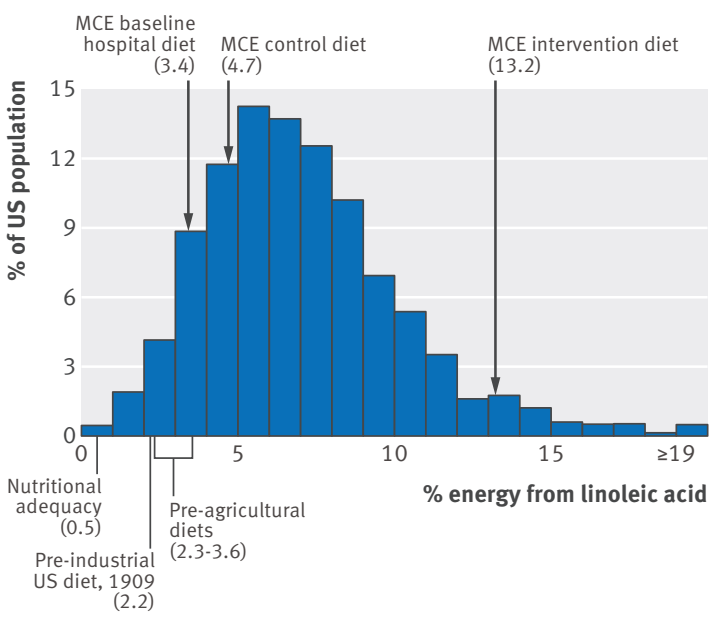

Fig 8 | Linoleic acid content of MCE diets compared with current and historical intakes in US in 2011-12 (NHANES, adults aged $\geq 20) .{ }^{58}$ Nutritional adequacy defined as lowest amount of dietary linoleic acid required to prevent deficiency symptoms. ${ }^{5960}$ Pre-agricultural diets modeled from fatty acid compositions of hunter-gatherer diets. ${ }^{56}$ Pre-industrial US diets calculated from US Department of Agriculture economic disappearance data ${ }^{61}$

benefit. Further, one way to interpret the unfavorable results of the two recovered trials is that high intakes of linoleic acid could have adverse effects in people who are prone to linoleic acid oxidation (such as smokers, heavy drinkers, and older adults).

\section{High linoleic acid intakes from vegetable oils are a} recent and atypical nutritional phenomenon

To interpret research on linoleic acid one needs to consider both the food sources and the amounts consumed. Individuals eating only minimally processed whole foods-as everyone did until about 100 years agowould have consumed about 2-3\% of calories from linoleic acid ${ }^{567}$ (fig 8). By contrast, among industrialized populations today, most linoleic acid intake is derived from highly concentrated vegetable oils, in which the fatty acids are separated from the fiber, protein, and micronutrients that are naturally present in vegetables

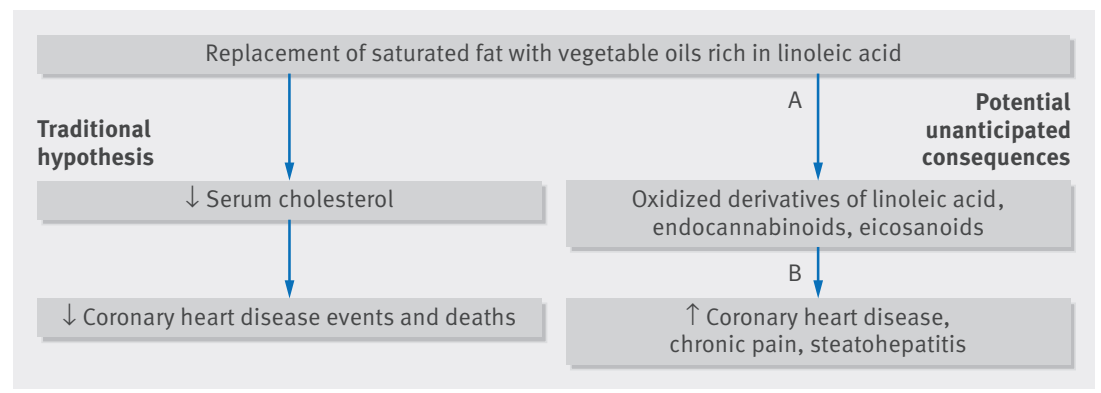

Fig 9 | Potential unanticipated consequences of high linoleic acid intake. $A=$ increased consumption of vegetable oils rich in linoleic acid alters non-cholesterol lipid mediators, including hydroperoxy- and hydroxy-octadecadienoic acids, eicosanoids, and endocannabinoids; $B$ =hydroperoxy- and hydroxy-octadecadienoic acids have been linked to coronary heart disease pathogenesis via mechanisms independent of traditional diet-heart hypothesis; non-cholesterol lipid mediators can also contribute to development of other common conditions including chronic pain and steatohepatitis and seeds ${ }^{61}$ (table $\mathrm{H}$, appendix). Because these concentrated sources of linoleic acid are used widely as cooking and frying oils and added to many processed and packaged food items, the linoleic acid content of modern industrialized diets is much higher than natural diets (fig 8). For example, mean linoleic acid intake in the US of about $17 \mathrm{~g}$ a day ( $7 \%$ of calories) $)^{58}$ is much higher than the approximately $6 \mathrm{~g}$ of daily linoleic acid provided by natural food diets without added vegetable oils. If these concentrated sources are considered to be dietary supplements, on average Americans ingest the equivalent of 11 capsules of $1 \mathrm{~g}$ linoleic acid a day above and beyond intake from natural foods.

\section{Impacts of high linoleic acid intake extend beyond serum cholesterol lowering}

Increasing dietary linoleic acid has been shown to increase oxidized linoleic acid derivatives in a dosedependent manner in many tissues. ${ }^{4062-66}$ These oxidized derivatives, along with other non-cholesterol lipid mediators, have been implicated in the pathogenesis of many diseases including coronary heart disease, ${ }^{14}$ chronic pain, ${ }^{4067-69}$ and steatohepatitis ${ }^{70-72}$ (fig 9). While the biochemical and clinical consequences of high intakes are incompletely understood, there is a possibility for unintended harm. These potential risks highlight the importance of ensuring that the full evidence base from randomized controlled trials is available for consideration by scientists, policymakers, and the public.

\section{Historical context for publication of Sydney Diet Heart Study and MCE findings}

With today's recognition of publication bias and requirements for trial registration and timely publication on completion of registered trials, the omission of key results of these two trials from the literature might seem difficult to understand. In the case of the MCE, the crude study results were clearly at odds with prevailing beliefs. One can speculate that the investigators and sponsors would have wanted to distinguish between a failed theory and a failed trial before publication. While robustly designed and carefully executed, the MCE had several unique features that complicated analysis and could have biased results. The MCE investigators might have been concerned that heavy censoring or the complicated health and social histories of study participants could have impacted results. In addition, the methods of adjusting survival time analyses for covariates were just emerging, and statistical software packages were not widely available, even at the time the Broste thesis was written. Failure to measure cholesterol concentrations for participants who left the hospital before one year could have introduced bias and would have reduced power for some analyses, and the heavy censoring might have further contributed to the possibility of type II errors. There would have been little or no scientific or clinical trial literature at the time to support findings that were so contrary to prevailing beliefs and public policy. And, finally, it is possible that medical journal reviewers would not have accepted study results for the reasons cited above. 
Whatever the explanation for key MCE data not being published, there is growing recognition that incomplete publication of negative or inconclusive results can contribute to skewed research priorities and public health initiatives. ${ }^{73-75}$ Recovery of unpublished data can alter the balance of evidence and, in some instances, can lead to reversal of established policy or clinical practice positions. ${ }^{73}$ Figure 10 provides a historical context for the completion and publication of the MCE and Sydney Diet Heart Study results in relation to key US policy events over the past half century. It is interesting to speculate whether complete publication of randomized controlled trial results might have altered key policy decisions promoting replacement of saturated fat with linoleic acid rich oils (such as the 1977 McGovern report ${ }^{76}$ and National Cholesterol Education Program (1984-85) ${ }^{45}$ ) or contributed to a shift in research priorities.

Implications for recovery of additional MCE data files MCE data files that remain missing could provide further insights into the diet-heart hypothesis (table D in appendix). For example, there is a suggestion that high linoleic acid intake could adversely affect those who are known to have increased linoleic acid oxidation, including smokers, heavy drinkers, those with established coronary heart disease, and older adults. ${ }^{14}$ With additional data recovery one could determine whether the high linoleic acid diet had deleterious effects in

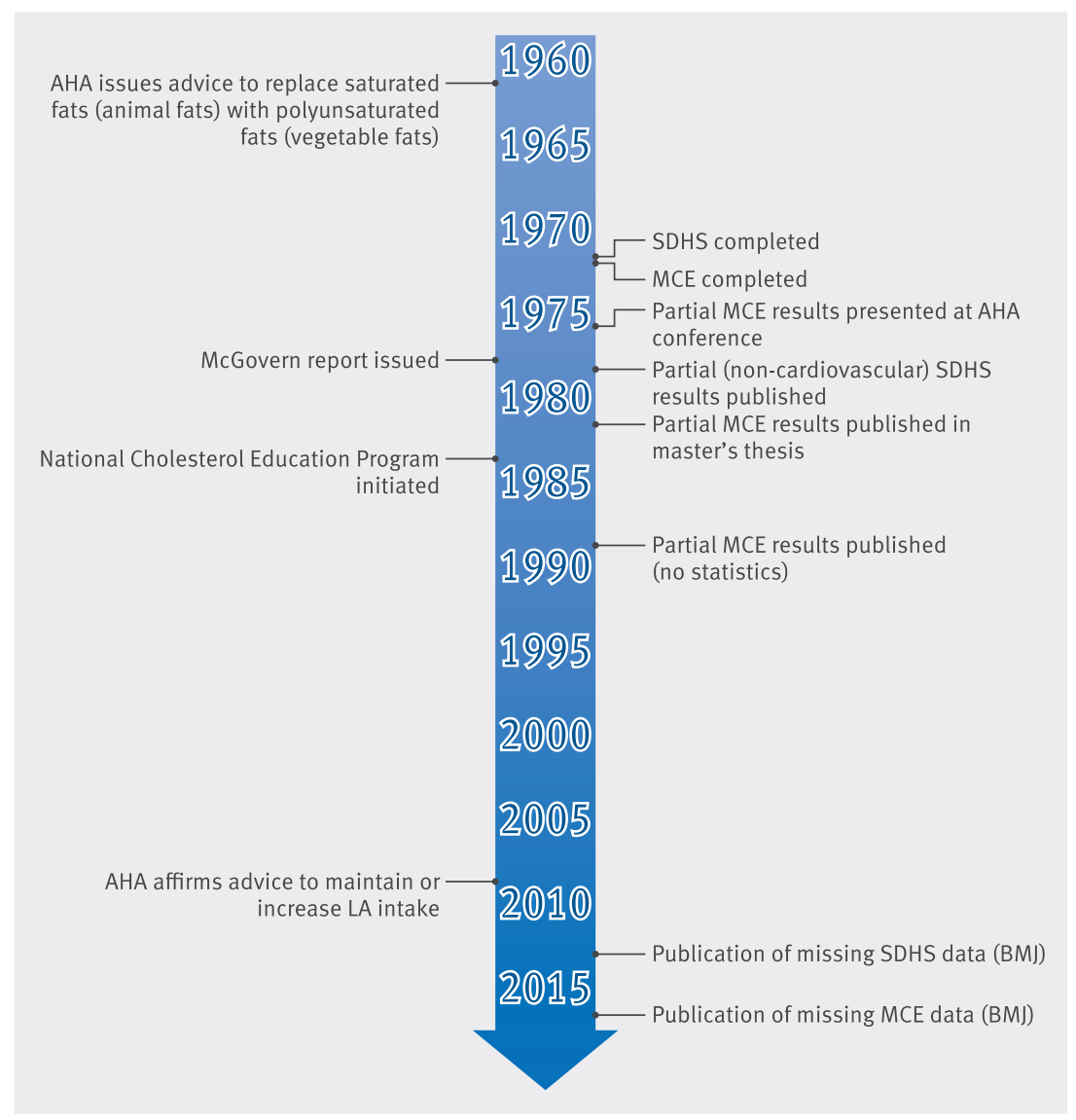

Fig 10 | Diet-heart timeline: key research and policy events. SDHS=Sydney Diet heart Study; MCE=Minnesota Coronary Experiment; $\mathrm{AHA}=$ American Heart Association; $\mathrm{LA}=$ linoleic acid these subgroups. The partial recovery of 149 heart and aorta autopsy files provides an intriguing clue that the intervention might have had unfavorable effects. As 146 heart and aorta files and the data on the full cohort of 295 autopsied brains remain missing, however, one cannot draw conclusions from these provisional findings. It is highly unlikely that a diet-heart trial of the size and scope of the MCE will ever be conducted again so it is essential that these missing autopsy files are recovered and analyzed as specified in the 1967 MCE grant application and FORTRAN coding sheets. ${ }^{619-21}$

\section{Strengths and limitations}

The MCE had several exceptional features and strengths. For example, the MCE is by far the largest randomized controlled trial to test the central diet-heart tenet that lowering serum cholesterol by replacing saturated fat with linoleic acid rich vegetable oil will translate to a lower risk of coronary heart disease and death. The MCE is also the only randomized controlled trial to test the clinical effects of increasing linoleic acid in large cohorts of women and individuals aged $\geq 65$ and the only such trial to complete a postmortem assessment of atherosclerosis grade and infarct status.

As MCE participants were randomly assigned and all meals were provided, we know that changes in linoleic acid and saturated fat were from the diets provided. Thus, MCE effectively dealt with the problem of healthy consumer bias that confounds many observational studies. ${ }^{31}$ Other strengths were that the intervention effectively lowered serum cholesterol and that these changes were consistent with predictions based on the Keys equation and the chemical analysis of study foods.

The MCE also had several important limitations in study design and generalizability. Participants were followed only while in hospital, and only about a quarter of randomized participants remained in the study for a year or longer. Although the original investigators emphasized this subsample and believed the longer follow-up to be more informative, it is a limitation that the association between serum cholesterol and death can now be examined only among those who survived the first year and remained in hospital. Moreover, even though we used data from a randomized controlled trial, the analysis of the association between serum cholesterol and death is observational in nature. Therefore, it is not possible to examine causality or to disentangle changes in serum cholesterol because of diet from changes because of other factors. In addition, low density lipoprotein (and high density lipoprotein) subfractions, which are more closely linked to risk of coronary heart disease than total serum cholesterol, were not assessed. Numerous randomized controlled trials, however, have shown that replacing saturated fat with vegetable oil rich in linoleic acid leads to predictable lowering of low density lipoprotein without altering high density lipoprotein. ${ }^{8}$ Thus, the serum cholesterol lowering effects of the MCE diets were likely specific to low density lipoprotein.

Because the trans fatty acid contents of MCE study diets are not available, one could speculate that the lack of benefit in the intervention group was because of 
increased consumption of trans fat. Indeed, in addition to liquid corn oil the intervention diet also contained a serum cholesterol lowering soft corn oil polyunsaturated margarine, which likely contained some trans fat. The MCE principal investigator (Ivan Frantz) and co-principal investigator (Ancel Keys), however, were well aware of the cholesterol raising effects of trans fat prior to initiating the MCE. ${ }^{77}$ Moreover, Frantz and Keys previously devised the diets used in the institutional arm of the National Diet Heart Feasibility Study (NDHS), which achieved the greatest reductions in serum cholesterol of all NDHS study sites. ${ }^{2}$ Hence, it is highly likely that this experienced MCE team selected products containing as little trans fat as possible to maximize the achieved degree of cholesterol lowering. Perhaps more importantly, it is clear from the MCE grant proposal that common margarines and shortenings (major sources of trans fat) were important components of the baseline hospital diets and the control diet (but not the intervention diet). Thus, confounding by dietary trans fat is an exceedingly unlikely explanation for the lack of benefit of the intervention diet.

Another limitation in the interpretation is incomplete data recovery. For example, in the Sydney Diet Heart Study the increased mortality in the high linoleic acid group was most evident in smokers and heavy drinkers. ${ }^{14}$ Without additional recovery of MCE data we are not able to determine if the effects of the high linoleic acid diet varied by smoking status, pre-existing coronary heart disease, psychiatric history, or drug use. Also, as we were not able to recover data for the full MCE cohort, we present the Broste thesis as recovered, without a more thorough analysis of differences in mortality between groups.

\section{Limitations in generalizability}

The MCE intervention diet contained almost twice as a much linoleic acid as the average American diet. Only a small percentage of the US population currently consumes linoleic acid in amounts that overlap those in the MCE intervention diet (fig 8). As this high linoleic acid diet produced a maximum lowering of serum cholesterol, it was ideal for testing the diet-heart tenet that serum cholesterol is the critical mediator linking diet to coronary heart disease. However, one cannot necessarily extrapolate findings to lower linoleic acid intakes.

The decision to conduct the MCE in mental hospitals and nursing homes reduced the number of missed meals and maximized the achieved degree of serum cholesterol lowering. However, the results are not necessarily generalizable to populations without mental illnesses or living outside nursing homes. As the MCE, Sydney Diet Heart Study, and other diet-heart trials used concentrated vegetable oils, the results should not be generalized to nuts or other unprocessed foods containing linoleic acid.

\section{Meta-analysis limitations}

Limitations of our meta-analysis include the small number of randomized controlled trials that have tested the effects of replacing saturated fat with linoleic acid rich oil, the differences in design and population characteristics of each trial, and the many limitations of meta-analyses in general (appendix part 2).

\section{The Big Picture}

The molecules that we eat every day as foods act as substrates, which enter into and regulate numerous highly leveraged biochemical pathways. 253840697879 Thus, although the story of the traditional diet-heart hypothesis did not unfold as predicted, the foods that we eat likely play critical roles in the pathogenesis of many diseases. Given the complexity of biological systems and limitations of our research methods, however, current understanding of the biochemical and clinical effects of foods is rudimentary. The history of the traditional diet-heart hypothesis suggests that nutrition research could be improved by not overemphasizing intermediate biomarkers; cautious interpretation of non-randomized studies; and ensuring timely and complete publication of all randomized controlled trials. Given the limitations of current evidence, the best approach might be one of humility, highlighting limitations of current knowledge and setting a high bar for advising intakes beyond what can be provided by natural diets.

\section{Summary and conclusion}

Available evidence from randomized controlled trials shows that replacement of saturated fat with linoleic acid effectively lowers serum cholesterol but does not support the hypothesis that this translates to a lower risk of death from coronary heart disease or all causes. MCE findings add to growing evidence that incomplete publication has contributed to overestimation of benefits, and underestimation of potential risks, of replacing saturated fat with vegetable oils rich in linoleic acid.

We thank the original MCE team of researchers for their contributions, including Ivan Frantz (principal investigator), Ancel Keys (co-principal investigator), Patricia Ashman (senior nutritionist and administrative assistant), Gerald Lee (physician assistant), Paul Lober (pathologist), Lael Gatewood (statistician), Sandra Knapp (statistical clerk), the staff of the seven Minnesota hospitals, and all the patients who participated in the study. We also thank the following people for their contribution to the present manuscript: John Svee (data conversion resource, Westminster, CO, US) for the conversion of 9-track tape data and greenbar data to modern spreadsheet format; Toni Calzone for expertise in optical character recognition and magnetic tape data recovery; Bradley Kuennen and Mathew Schuler (Iowa State University Special Collections Department), Rachael Davis (E G Miner Library), National Library of Medicine staff, and Margo Denke for efforts searching for study data and materials; Alan Hoofring (Intramural NIH Medical Arts Design Section) for expertise in medical art; Sarah Strandjord for general research assistance; Denisse Zamora for expertise in graphic design; and Mark S Horowitz for expertise in statistical programming and technical writing.

Contributors: CER and DZ contributed as co-first authors. CER designed and directed the project; located, managed, and validated the recovered data and materials; performed literature review; and was the main writer of the manuscript. DZ conducted the statistical analyses and was a main writer of the manuscript. SFMH located, managed and validated the recovered data, and assisted in the literature review and in writing and revising the manuscript. KRF conducted the systematic review and meta-analyses, in collaboration with SFMH, CER, DZ, JMD, and CMS. SKB wrote the 1981 master's thesis, provided insights into trial design, data analyses and incomplete publication, and contributed to the writing and revision of the manuscript. RPF located recovered data, wrote the tribute to Ivan Frantz and the MCE research team (in appendix), and revised the manuscript. JMD, CMS, and KRF contributed to the statistical analysis, interpretation of study results, and the writing and revision of the 
manuscript. AR validated the recovered data and revised the manuscript. JRH directed the project and contributed to writing and revision of the manuscript. All authors contributed to analyses or interpretation of results and to the intellectual content of the manuscript. CER is guarantor.

Funding: The MCE was funded by the US Public Health Service and the National Heart Institute through the R01 mechanism (grant HE09686). The intramural program of the National Institute on Alcohol Abuse and Alcoholism, National Institutes of Health, and the University of North Carolina Program on Integrative Medicine (National Institutes of Health grant T-32 AT003378) supported data recovery and evaluation. The content is solely the responsibility of the authors and does not necessarily represent the official views of the National Institutes of Health or the US Public Health Service.

Competing interests: All authors have completed the ICMJE uniform disclosure form at www.icmje.org/coi_disclosure.pdf and declare: support from the Intramural Program of the National Institute on Alcohol Abuse and Alcoholism for the submitted work; no financial relationships with any organizations that might have an interest in the submitted work in the previous three years; no other relationships or activities that could appear to have influenced the submitted work.

Ethical approval: The MCE was approved by the clinical research committee of the University of Minnesota and by each of the seven collaborating hospitals.

Data sharing: Dataset requests should be sent to the corresponding author. Data sharing consent was not obtained, but the presented data are anonymized and risk of identification is low. Please contact D Zamora at zamora@unc.edu for questions about statistical code.

Transparency: The manuscript's guarantor affirms that the manuscript is an honest, accurate, and transparent account of the study being reported; that no important aspects of the present data recovery and analysis have been omitted; and that any discrepancies from the study as planned have been explained.

This is an Open Access article distributed in accordance with the Creative Commons Attribution Non Commercial (CC BY-NC 3.0) license, which permits others to distribute, remix, adapt, build upon this work non-commercially, and license their derivative works on different terms, provided the original work is properly cited and the use is noncommercial. See: http://creativecommons.org/licenses/by-nc/3.0/.

1 Sherwin R. Controlled trials of the diet-heart hypothesis: some comments on the experimental unit. Am J Epidemiol 1978;108:92-9.

2 Executive Committee on Diet and Heart Disease. National Diet-Heart Study Report. American Heart Association, 1968.

3 Getz GS, Vesselinovitch D, Wissler RW. A dynamic pathology of arteriosclerosis. Am J Med 1969;46:657-73. doi:10.1016/0002-9343(69)90018-7.

4 Camejo G, Waich S, Quintero G, Berrizbeitia ML, Lalaguna F. The affinity of low density lipoproteins for an arterial macromolecular complex. A study in ischemic heart disease and controls. Atherosclerosis 1976;24:341-54. doi:10.1016/0021-9150(76)90126-X.

5 Armstrong ML, Warner ED, Connor WE. Regression of coronary atheromatosis in rhesus monkeys. Circ Res 1970;27:59-67. doi:10.1161/01.RES.271.59.

6 Frantz ID Jr, Keys A. R01 HE 0986-03 Research Grant Application: Effect of a Dietary Change on Human Cardiovascular Disease "The Minnesota Coronary Survey”. 1967.http://www.add.lib.iastate.edu/ spcl/manuscripts/MS576.html

7 Broste S. Lifetable Analysis of the Minnesota Coronary Survey. University of Minnesota, 1981

8 Mensink RP, Zock PL, Kester AD, Katan MB. Effects of dietary fatty acids and carbohydrates on the ratio of serum total to HDL cholesterol and on serum lipids and apolipoproteins: a meta-analysis of 60 controlled trials. Am J Clin Nutr 2003;77:1146-55.

9 Van Assche K, Capitaine L, Pennings G, Sterckx S. Governing the postmortem procurement of human body material for research. Kennedy Inst Ethics / 2015;25:67-88. doi:10.1353/ken.2015.0000.

10 Keys A, Anderson IT, Grande F. Prediction of serum-cholesterol responses of man to changes in fats in the diet. Lancet 1957;273:95966. doi:10.1016/S0140-6736(57)91998-0.

11 Keys A, Anderson JT, Grande F. Serum cholesterol response to changes in the diet: IV. Particular saturated fatty acids in the diet. Metabolism 1965;14:776-87. doi:10.1016/0026-0495(65)90004-1.

12 Keys A, Anderson JT, Grande F. Serum cholesterol response to changes in the diet: I. Iodine value of dietary fat versus 2S-P. Metabolism 1965;14:747-58. doi:10.1016/0026-0495(65)90001-6.

13 Caplan AL. Morality dissected: a plea for reform of current policies with respect to autopsy. Hum Pathol 1984;15:1105-6. doi:10.1016/ S0046-8177(84)80304-4.
14 Ramsden CE, Zamora D, Leelarthaepin B, et al. Use of dietary linoleic acid for secondary prevention of coronary heart disease and death: evaluation of recovered data from the Sydney Diet Heart Study and updated meta-analysis. BMJ 2013;346:e8707. doi:10.1136/bmj.e8707.

15 Frantz ID Jr, Dawson EA, Ashman PL, et al. Test of effect of lipid lowering by diet on cardiovascular risk. The Minnesota Coronary Survey. Arteriosclerosis 1989;9:129-35. doi:10.1161/01.ATV.9.1.129.

16 Brewer ER, Ashman PL, Kuba K. The Minnesota Coronary Survey: composition of their diets, adherence, and serum lipid response. Circulation 1975:51(suppl 2):1-269.

17 Dawson E, Gatewood L. The Minnesota coronary survey: methodology and characteristics of the population. Circulation 1975;52(suppl 2):271.

18 Frantz ID, Dawson EA, Kuba K, Brewer ER, Gatewood LC, Bartsch GE. The Minnesota Coronary Survey: Effect of diet on cardiovascular events and deaths. American Heart Association Scientific Proceedings. 1975 October 51 \& 52(Supplement II): II-4

19 Frantz ID. (PI) R01 HE 0986-02 Supplementary Progress Report: Effect of a Dietary Change on Human Cardiovascular Disease "The Minnesota Coronary Survey”, August 31, 1967. http://www.add.lib. iastate.edu/spcl/manuscripts/MS576.html

20 Frantz ID, Jr. R01 HE 0986-02 Second Supplementary Progress Report: Effect of a Dietary Change on Human Cardiovascular Disease "The Minnesota Coronary Survey", September 25, 1967.http://www.add.lib. iastate.edu/spcl/manuscripts/MS576.html

21 Spiekerman RE, Brandenburg JT, Achor RW, Edwards JE. The spectrum of coronary heart disease in a community of 30,000. A clinicopathologic study. Circulation 1962;25:57-65. doi:10.1161/01.CIR.25.1.57.

22 Frantz I. Chemical Analysis of Three Week Food Collection in the Control and Treatment Diets for the Minnesota Coronary Survey: Unpublished Data Comparing the Institutional BC Diets at the Seven Individual Hospitals of the MCS. 1971.http://www.add.lib.iastate.edu/ spcl/manuscripts/MS576.htm

23 Moher D, Liberati A, Tetzlaff], Altman DG. PRISMA Group. Preferred reporting items for systematic reviews and meta-analyses: the PRISMA statement. PLoS Med 2009;6:e1000097. doi:10.1371/journal. pmed.1000097.

24 Enig MG, Atal S, Keeney M, Sampugna J. Isomeric trans fatty acids in the U.S. diet[eng.]. J Am Coll Nutr 1990;9:471-86. doi:10.1080/07315724.1990.10720404

25 Sastry PS. Lipids of nervous tissue: composition and metabolism. Prog Lipid Res 1985;24:69-176. doi:10.1016/0163-7827(85)90011-6.

26 DHEW Publication (NIH) 75-628: Manual of Laboratory OperationsLipid Research Clinic Program, Volume 1- Lipid and Lipoprotein Analysis, May 1974

27 Keys A, Grande F, Anderson JT. Bias and misrepresentation revisited: "perspective" on saturated fat. Am / Clin Nutr 1974;27:188-212.

28 Bandeen-Roche K, Xue QL, Ferrucci L, et al. Phenotype of frailty: characterization in the women's health and aging studies. J Gerontol A Biol Sci Med Sci 2006;61:262-6. doi:10.1093/gerona/61.3.262.

29 Fried LP, Tangen CM, Walston J, et al. Cardiovascular Health Study Collaborative Research Group. Frailty in older adults: evidence for a phenotype. J Gerontol A Biol Sci Med Sci 2001;56:M146-56. doi:10.1093/gerona/56.3.M146.

30 Rockwood MR, Howlett SE. Blood pressure in relation to age and frailty. Can Geriatr J 2011;14:2-7. doi:10.5770/cgj.v14i1.1.

31 Maki KC, Slavin JL, Rains TM, Kris-Etherton PM. Limitations of observational evidence: implications for evidence-based dietary recommendations. Adv Nutr 2014;5:7-15. doi:10.3945/an.113.004929.

32 Young SS, Karr A. Deming, data and observational studies: A process out of control and needing fixing. Significance 2011;8:116-20 doi:10.1111/j.1740-9713.2011.00506.x.

33 Kristal AR, Peters U, Potter JD. Is it time to abandon the food frequency questionnaire? Cancer Epidemiol Biomarkers Prev 2005;14:2826-8. doi:10.1158/1055-9965.EPI-12-ED1.

34 Stampfer MJ, Colditz GA. Estrogen replacement therapy and coronary heart disease: a quantitative assessment of the epidemiologic evidence. Prev Med 1991:20:47-63. doi:10.1016/0091-7435(91)90006-P.

35 Nelson HD, Humphrey LL, Nygren P, Teutsch SM, Allan JD. Postmenopausal hormone replacement therapy: scientific review. JAMA 2002;288:872-81. doi:10.1001/jama.288.7.872

36 Grodstein F. Invited commentary: can selection bias explain the cardiovascular benefits of estrogen replacement therapy? Am J Epidemiol 1996;143:979-82, discussion 983-4. doi:10.1093/ oxfordjournals.aje.a008679.

37 Oh K, Hu FB, Manson JE, Stampfer MJ, Willett WC. Dietary fat intake and risk of coronary heart disease in women: 20 years of follow-up of the nurses' health study[eng.]. Am J Epidemiol 2005;161:672-9. doi:10.1093/aje/kwi085.

38 Vangaveti V, Baune BT, Kennedy RL. Hydroxyoctadecadienoic acids: novel regulators of macrophage differentiation and atherogenesis. Ther Adv Endocrinol Metab 2010;1:51-60. doi:10.1177/2042018810375656.

39 Farvid MS, Ding M, Pan A, et al. Dietary linoleic acid and risk of coronary heart disease: a systematic review and meta-analysis of prospective cohort studies. Circulation 2014:130:1568-78. doi:10.1161/CIRCULATIONAHA.114.010236. 
40 Ramsden CE, Ringel A, Majchrzak-Hong SF, et al. Dietary linoleic acid-induced alterations in pro- and anti-nociceptive lipid autacoids: Implications for idiopathic pain syndromes? Mol Pain (in press).

41 Chowdhury R, Warnakula S, Kunutsor S, et al. Association of dietary, circulating, and supplement fatty acids with coronary risk: a systematic review and meta-analysis. Ann Intern Med 2014;160:398406. doi:10.7326/M13-1788

42 de Oliveira Otto MC, Wu JH, Baylin A, et al. Circulating and dietary omega-3 and omega- 6 polyunsaturated fatty acids and incidence of CVD in the Multi-Ethnic Study of Atherosclerosis. / Am Heart Assoc 2013;2:e000506. doi:10.1161/JAHA.113.000506.

$43 \mathrm{Wu}$ JH, Lemaitre RN, King IB, et al. Circulating omega-6 polyunsaturated fatty acids and total and cause-specific mortality: the Cardiovascular Health Study. Circulation 2014;130:1245-53. doi:10.1161/CIRCULATIONAHA.114.011590

44 Willett WC. The role of dietary n-6 fatty acids in the prevention of cardiovascular disease. J Cardiovasc Med (Hagerstown) 2007;8(Suppl 1):S42-5. doi:10.2459/01.JCM.0000289275.72556.13.

45 Lowering Blood Cholesterol To Prevent Heart Disease. NIH Consensus Statement Online 1984 Dec 10-12; 5(7):1-11.

46 Ramsden CE, Zamora D, Leelarthaepin B, et al. Use of dietary linoleic acid for secondary prevention of coronary heart disease and death: evaluation of recovered data from the Sydney Diet Heart Study and updated meta-analysis. BM/ 2013:346:e8707.http://www.bmj.com/ content/346/bmj.e8707/rr/631590doi:10.1136/bmj.e8707.

47 Ramasamy I. Recent advances in physiological lipoprotein metabolism. Clin Chem Lab Med 2014;52:1695-727. doi:10.1515/ cclm-2013-0358.

48 He J, Lee JH, Febbraio M, Xie W. The emerging roles of fatty acid translocase/CD36 and the aryl hydrocarbon receptor in fatty liver disease. Exp Biol Med (Maywood) 2011;236:1116-21. doi:10.1258/ ebm.2011.011128.

49 Diffenderfer MR, Schaefer E). The composition and metabolism of large and small LDL. Curr Opin Lipidol 2014:25:221-6. doi:10.1097/ MOL.0000000000000067.

50 Spady DK. Hepatic clearance of plasma low density lipoproteins. Semin Liver Dis 1992:12:373-85. doi:101055/s-2008-1040407.

51 Naci H, Brugts JJ, Fleurence R, Tsoi B, Toor H, Ades AE. Comparative benefits of statins in the primary and secondary prevention of major coronary events and all-cause mortality: a network meta-analysis of placebo-controlled and active-comparator trials. Eur J Prev Cardiol 2013;20:641-57. doi:10.1177/2047487313480435

52 Buchwald H, Varco RL, Matts JP, et al. Effect of partial ileal bypass surgery on mortality and morbidity from coronary heart disease in patients with hypercholesterolemia. Report of the Program on the Surgical Control of the Hyperlipidemias (POSCH). N Engl / Med 1990;323:946-55. doi:10.1056/NEIM199010043231404

53 The Coronary Drug Project. The Coronary Drug Project. Findings leading to discontinuation of the 2.5-mg day estrogen group. The coronary Drug Project Research Group. JAMA 1973;226:652-7. doi:10.1001/jama.1973.03230060030009.

54 The Coronary Drug Project. The Coronary Drug Project. Initial findings leading to modifications of its research protocol. JAMA 1970:214:1303-13. doi:10.1001/jama.1970.03180070069012.

55 The coronary drug project. Findings leading to further modifications of its protocol with respect to dextrothyroxine. The coronary drug project research group. JAMA 1972;220:996-1008. doi:10.1001/ jama1972.03200070084015.

56 Kuipers RS, Luxwolda MF, Dijck-Brouwer DA, et al. Estimated macronutrient and fatty acid intakes from an East African Paleolithic diet[eng.]. Br J Nutr 2010;104:1666-87. doi:10.1017/ S0007114510002679.

57 Blasbalg TL, Hibbeln JR, Ramsden CE, Majchrzak SF, Rawlings RR. Changes in consumption of omega-3 and omega- 6 fatty acids in the United States during the 20th century. Am J Clin Nutr 2011;93:950-62. doi:10.3945/ajen.110.006643.

58 US Department of Agriculture, Agricultural Research Service. Nutrient Intakes from Food: Mean Amounts Consumed per Individual, by Gender and Age, What We Eat in America, National Health and Nutrition Examination Survey (NHANES 2011-2012).

59 Guesnet P, Lallemand SM, Alessandri JM, Jouin M, Cunnane SC. $\alpha$-Linolenate reduces the dietary requirement for linoleate in the growing rat. Prostaglandins Leukot Essent Fatty Acids 2011;85:35360. doi:10.1016/j.plefa.2011.08.003.
60 Blanchard H, Pédrono F, Boulier-Monthéan N, Catheline D, Rioux V, Legrand P. Comparative effects of well-balanced diets enriched in $\alpha$-linolenic or linoleic acids on LC-PUFA metabolism in rat tissues. Prostaglandins Leukot Essent Fatty Acids 2013;88:383-9. doi:10.1016/j.plefa.2013.03.006.

61 US Department of Agriculture Agricultural Research Service. 2010. USDA National Nutrient Database for Standard Reference, Release 23. Nutrient Data Laboratory Home Page, http://www.ars.usda.gov/nutrientdata.

62 Ramsden CE, Ringel A, Feldstein AE, et al. Lowering dietary linoleic acid reduces bioactive oxidized linoleic acid metabolites in humans. Prostaglandins Leukot Essent Fatty Acids 2012;87:135-41. doi:10.1016/j.plefa.2012.08.004.

63 Marmesat S, Morales A, Velasco J, Carmen Dobarganes M. Influence of fatty acid composition on chemical changes in blends of sunflower oils during thermoxidation and frying. Food Chem 2012;135:2333-9. doi:10.1016/j.foodchem.2012.06.128.

64 Thompson LU, Aust R. Lipid Changes in French Fries and Heated Oils During Commercial Deep Frying and Their Nutritional and Toxicological Implications. Can Inst Food Sci Technol 1983;16:24653doi:10.1016/S0315-5463(83)72257-1.

65 Wilson R, Lyall K, Smyth L, Fernie CE, Riemersma RA. Dietary hydroxy fatty acids are absorbed in humans: implications for the measurement of 'oxidative stress' in vivo. Free Radic Biol Med 2002;32:162-8. doi:10.1016/S0891-5849(01)00780-8.

66 Ferreiro-Vera C, Priego-Capote F, Mata-Granados JM, Luque de Castro MD. Short-term comparative study of the influence of fried edible oils intake on the metabolism of essential fatty acids in obese individuals. Food Chem 2013;136:576-84. doi:10.1016/j.foodchem.2012.08.081.

67 Patwardhan AM, Akopian AN, Ruparel NB, et al. Heat generates oxidized linoleic acid metabolites that activate TRPV1 and produce pain in rodents. / Clin Invest 2010;120:1617-26. doi:10.1172//Cl41678.

68 Patwardhan AM, Scotland PE, Akopian AN, Hargreaves KM. Activation of TRPV1 in the spinal cord by oxidized linoleic acid metabolites contributes to inflammatory hyperalgesia. Proc Natl Acad Sci U S A 2009;106:18820-4. doi:10.1073/pnas.0905415106.

69 Ramsden CE, Faurot KR, Zamora D, et al. Targeted alteration of dietary n-3 and $\mathrm{n}-6$ fatty acids for the treatment of chronic headaches: a randomized trial. Pain 2013:154:2441-51. doi:10.1016/j.pain.2013.07.028.

70 Feldstein AE, Lopez R, Tamimi TA, et al. Mass spectrometric profiling of oxidized lipid products in human nonalcoholic fatty liver disease and nonalcoholic steatohepatitis. J Lipid Res 2010;51:3046-54. doi:10.1194/jlr.M007096

71 Kirpich IA, Feng W, Wang Y, et al. Ethanol and dietary unsaturated fat (corn oil/linoleic acid enriched) cause intestinal inflammation and impaired intestinal barrier defense in mice chronically fed alcohol. Alcohol 2013;47:257-64. doi:10.1016/j.alcohol.2013.01.005.

72 Liu H, Beier Jl, Arteel GE, et al. Transient receptor potential vanilloid 1 gene deficiency ameliorates hepatic injury in a mouse model of chronic binge alcohol-induced alcoholic liver disease. Am J Pathol 2015;185:43-54. doi:10.1016/j.ajpath.2014.09.007.

73 Godlee F, Loder E. Missing clinical trial data: setting the record straight. BMJ 2010;341:c5641. doi:10.1136/bmj.c5641

74 Lehman R, Loder E. Missing clinical trial data. BMJ 2012;344:d8158. doi:10.1136/bmi.d8158.

75 WHO Statement on Public Disclosure of Clinical Trial Results. WHO, 2015.

76 Dietary goals for the United States, 2d ed. prepared by the staff of the Select Committee on Nutrition and Human Needs, United States Senate. US Govt. Print. Office in Washington DC, 1977. https://openlibrary.org/ books/OL4373027M/Dietary_goals_for_the_United_States.

77 Anderson JT, Grande F, Keys A. Hydrogenated fats in the diet and lipids in the serum of man. J Nutr 1961;75:388-94.pmid:13861251.

78 Ramsden CE, Zamora D, Makriyannis A, et al. Diet-induced changes in $\mathrm{n}$-3- and n-6-derived endocannabinoids and reductions in headache pain and psychological distress. J Pain 2015;16:707-16. doi:10.1016/j. pain.2015.04.007.

79 Chemin J, Cazade M, Lory P. Modulation of T-type calcium channels by bioactive lipids. Pflugers Arch 2014;466:689-700. doi:10.1007/ s00424-014-1467-5.

Appendix: Supplementary material Appendix 2: MCE data collection forms 